\title{
Pressure effects on real-gas laminar counterflow
}

\author{
Albert Jordà Juanós ${ }^{\mathrm{a}, *}$, William A. Sirignano ${ }^{\mathrm{a}}$ \\ ${ }^{a}$ University of California, Irvine, CA 92697-3975
}

\begin{abstract}
Two important problems are studied: the combustion of hydrocarbons at higher pressures and the burning of hydrocarbon/water-vapor mixtures both of which are relevant to many applications including diesel combustion and hydrate fuels. To study both of these problems, a numerical analysis of a steady laminar methane-air counterflow diffusion flame at high pressure is presented. The mathematical model is based on the well-known similar solution for counterflow with special considerations given to the high density and to detailed transport and chemistry. Modifications of transport properties and associated time scales with increasing pressure are considered. Real gas behavior is examined through the use of a cubic equation of state and an enthalpy departure function. A more complete version of the energy equation is presented. The effects on flame structure, location, and peak temperature are analyzed for a range of pressure from 1-100 atm. Assessment of the different high-pressure corrections indicate that introduction of the cubic equation of state is the most profound adjustment, while the correction of transport properties is the least significant. The use of the enthalpy departure function is important. The flame structure and heat-release rate are confirmed to follow previously identified correlations with the pressure-weighted strain rate. Extinction occurs when the mass fraction of $\mathrm{H}_{2} \mathrm{O}$ vapor in the methane stream is greater than $67 \%$ and the mixture impinges against air. Small differences in results occur between the classical chemical equilibrium employing partial pressures versus the non-ideal formulation that uses fugacities. The Soret effect and radiative heat losses are shown to be negligible, even at the highest pressures. An order of magnitude analysis shows that turbulence generation is practically inconsequential.
\end{abstract}

Keywords: counterflow, diffusion, supercritical, methane hydrates, chemical equilibrium

\section{Introduction}

The present study will give similar solutions that provide reliable conclusions about the laminar steady axisymmetric counterflow diffusion flame structure and location at very high pressures. An application of these solutions is found, for example, in the flamelet model for turbulent flames. Combustion under high 5 pressures leads to an increase in both thermodynamic and fuel efficiencies as well as reduced emission of some pollutants. These advantages are causing the current trend towards burning at high-pressure regimes. For instance, aircraft gas turbine engines now operate at $\sim 30 \mathrm{~atm}$, a value that has been increasing at a near linear rate for more than 50 years and is expected to continue to do so [1. Another example is that of diesel engines, which obtain pressures as large as $\sim 60$ atm after ignition $[2-6]$. In many rocket engines, 10 one of the reactants is injected in a liquid form at a temperature which is below the critical value but at supercritical pressure. These conditions were named as transcritical 7 and the pressure takes values which are typically of the order of $100 \mathrm{~atm}$ but may reach levels as high as $400 \mathrm{~atm}[8] 10$.

Ideal-gas approximations are often considered in combustion problems with the argument that at high temperatures and low densities the compressibility factor will be close to unity. At very high pressures, however, this may not necessarily be true. It is the responsibility of any analyst treating these high-pressure

\footnotetext{
* Corresponding author

Email address: ajordaju@uci.edu (Albert Jordà Juanós)
} 
flows to justify the ideal-gas approximation. In our study, we present results for a canonical combustion configuration with both ideal and real-gas models for comparison purposes and error estimation.

Another motivation for the present study is combustion at high pressures where the fuel is a mixture of methane and water vapor in relation to gas hydrates. These materials are ice-like crystalline solids formed

20 from a network of hydrogen-bonded water molecules that encapsulate guest gas molecules 11. There is a significant amount of methane naturally stored in the form of methane hydrates, also known as methane ice or methane clathrates. Sediment containing methane hydrates is found within specific pressure-temperature conditions that occur in regions of permafrost and beneath the sea in outer continental margins at depths of the order of a thousand meters [12] where pressure reaches the value of $100 \mathrm{~atm}$. It is not in the scope of this

25 study to determine whether combustion of methane hydrates in their natural conditions is practical. Rather, it poses an interesting problem from which conclusions can be extracted for a broader range of applications, including rocket engines or exhaust gas recirculation (EGR) in engines. For a detailed description of methane hydrate combustion, the reader is referred to [13. Note that mixtures of fuel and water can appear in other situations, e.g., exhaust gas re-circulation, bio-fuels, and direct water injection.

The literature on counterflow diffusion flame problems is broad and continues to expand. It includes analytical, experimental and numerical approaches [14, 15. Such flames are of fundamental interest because they approximate a one-dimensional character and because residence times within the flame zone can be easily varied [16]. This laminar flame has also been proposed as a key element in the complex structure of turbulent nonpremixed flames [17].

Even though most of the literature deals with situations at atmospheric pressure and ideal-gas conditions, some recent papers used numerical methods to examine flame characteristics entailing real-gas formulations to account for the high-pressure effects. Also, most specific examples are for oxygen/hydrogen systems, with less attention being given to hydrocarbons. For instance, an $\mathrm{O}_{2} / \mathrm{H}_{2}$ system was explored to find a correlation between the flame thickness and heat-release rate with the pressure-weighted strain rate [18].

40 Flame instabilities were studied in [19] using a flamelet model. Detailed investigation of the extinction strain rates appeared later for counterflow diffusion flames at subcritical and supercritical conditions for oxygen/hydrogen mixtures 20] and for oxygen/n-alkane hydrocarbons [21. These papers identified general similarities in the mixture-fraction space in terms of flame temperature, species concentrations, flame thickness, and heat-release rate, suggesting that the flame behaviors at high pressure can be evaluated by their 45 counterpart at low pressure. With regard to the n-alkane family of fuels, similarities indicated that flame properties of a given hydrocarbon can be predicted from those of another hydrocarbon at the same flow conditions.

Counterflow diffusion flame experimental studies at atmospheric pressure are common. For instance, results of an axisymmetric configuration with methane and enriched air were compared to computational so solutions obtained from both one-dimensional and two-dimensional mathematical models [22. The comparison between the experimental and computational data yielded excellent agreement for all measured quantities. Studies of the same kind at elevated pressures are unconventional, but some exist. See, for example, 23 25] for pressures up to $2.5 \mathrm{MPa}$.

The literature also includes studies with regard to the presence of water premixed with the fuel stream 55 at atmospheric pressure. An experiment 26] was conducted under microgravity conditions to study the influence of water mists on premixed flames propagating in a cylindrical tube. Results showed that the flame speed initially increases due to the thermal expansion of combustion gases with higher water content, while a later decrease in flame speed is caused by heat removal, mixture dilution, and radical recombination. A numerical counterflow analysis was presented, where air was introduced on one side and a preheated mixture of water vapor/methane entered on the other side [27]. Extinction limits were explored and compared with experimental results. Agreement was found for suitable fuel and oxidizer flow rates. Extinction occurred with lower water content compared to experiments when simulations were performed at higher flow rates, revealing the influence of strain rate. In another study, the dilution extinction limits of $\mathrm{H}_{2}-\mathrm{O}_{2}$ diffusion flames were assessed by mixing water with the fuel, where $\mathrm{H}_{2} \mathrm{O}$ modeled combustion products [28]. Other reasons for water injection into the combustion chamber include decreasing exhaust temperature and associated $\mathrm{NOx}$ emissions, flame extinguishment, or studying combustion efficiency [29]32].

In comparison with the reviewed literature, the current study contributes in several significant new 
aspects: (1) a real-gas model that entails fewer simplifying assumptions than previous studies (a more complete energy equation with the associated enthalpy correction for mixtures is presented and evaluated); (2)

70 evaluation of the effects of various high-pressure corrections and determination of their relative importance (3) evaluation of the correct use of fugacities as opposed to partial pressures in chemical-equilibrium calculations; (4) quantification of the radiative heat losses at high pressure; (5) evaluation of the Soret effect at high pressure; (6) analysis of turbulent kinetic energy generation due to shear in the stagnation plane; (7) extension of scaling rules to higher pressures; and (8) analysis of the effects of introducing water vapor in 75 the fuel stream at high pressure. To the best of the authors' knowledge, points 1 through 7 have not been done before. Point 8 has been done for a $\mathrm{H}_{2}-\mathrm{O}_{2}$ flame [28, but not for methane and air at high pressures in relation to the methane hydrates and other applications.

In Section 2, we review the mathematical model that we use in our study. The equation of state is discussed in Section 3. The method of solution, validation, and results are presented in Sections 4, 5, and 6. respectively. Supporting information on chemical equilibrium is presented in Appendix A

\section{Model}

Figure 1 shows a sketch that represents the axisymmetric flow between two circular opposed jets and the flame that is generated. Pure methane or a mixture of methane with water vapor flows from the left nozzle while air flows from the right nozzle. Under the boundary-layer approximation, the two-dimensional problem may be simplified to a system of ODEs with the independent variable that is the x-coordinate normal to the stagnation plane [33, 34. Slightly different formulations are available, depending on which parameter is chosen to alter the strain. Prescribing the momentum fluxes at the exit of the nozzles fixes the radial pressure gradient and the strain rate of the problem, or vice versa. The approach described in [34] is followed with modifications in the energy equation and the equation of state.

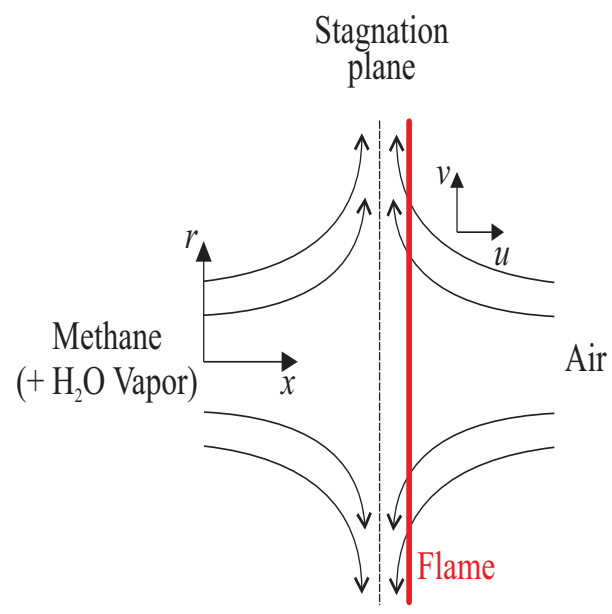

Figure 1: Non-premixed counterflow axisymmetric configuration.

The governing equations are summarized below, starting with the steady-state continuity equation in cylindrical coordinates.

$$
\frac{\partial(r \rho u)}{\partial x}+\frac{\partial(r \rho v)}{\partial r}=0
$$

90 The stream function is introduced as $\Psi(x, r) \equiv r^{2} F(x)$, which satisfies the steady-state continuity equation exactly if

$$
\frac{\partial \Psi}{\partial r}=r \rho u=2 r F \quad \text { and } \quad-\frac{\partial \Psi}{\partial x}=r \rho v=-r^{2} \frac{d F}{d x}
$$


Then, the axial velocity $u$ depends only on $x$ and the radial velocity $v$ varies linearly in $r$. The temperature $T$ and species mass fractions $Y_{k}$ are also functions of $x$ only. Using the small Mach number approximation, the thermodynamic pressure $p$ is assumed to be constant in the equation of state but pressure-gradient 95 terms are allowed in the momentum equations, where the term $d\left(r \tau_{r r}\right) / d r$ associated with the stress in the radial direction is neglected from the boundary-layer analysis. The pressure gradient in the axial direction is zero at the stagnation point, even without taking any boundary-layer approximation. Furthermore, the Navier-Stokes equations are satisfied exactly at that point. This is a classic result for stagnation-point flow. Away from the stagnation point, but still in the central streamline, the pressure gradient is of the order of Mach number squared $\left(M^{2}\right)$. Also, we have that $M<<1$, given that the flow speed will be at most $1 \mathrm{~m} / \mathrm{s}$, and the speed of sound will be around $340 \mathrm{~m} / \mathrm{s}$ in the worst case scenario. Therefore, the pressure gradient in the axial direction is negligible, and the stagnation pressure, which will be the same on either side of the stagnation plane, will also be essentially the same at the boundaries.

With these considerations and neglect of body forces, the momentum equations reduce to third-order ordinary differential equations. From these equations, an eigenvalue independent of $x$ appears: $H=r^{-1} d p / d r$.

Define the function $G(x)=d F / d x$. Then, the radial momentum equation becomes a 2nd-order ODE rather than one of 3 rd order,

$$
H-2 \frac{d}{d x}\left(\frac{F G}{\rho}\right)+\frac{3}{\rho} G^{2}+\frac{d}{d x}\left[\mu \frac{d}{d x}\left(\frac{G}{\rho}\right)\right]=0
$$

where $\mu$ is the viscosity.

The species continuity equation is

$$
2 F \frac{d Y_{k}}{d x}+\frac{d}{d x}\left(\rho Y_{k} V_{k}\right)-W_{k} \dot{\omega}_{k}=0 \quad k=1,2, \ldots, K
$$

where the index $k$ identifies each species and $K$ is the total number of species.

The energy equation for non-ideal gases is

$$
2 F\left(\frac{d h}{d x}-\sum_{k=1}^{K} h_{k} \frac{d Y_{k}}{d x}\right)-\frac{d}{d x}\left(\lambda \frac{d T}{d x}\right)+\rho \sum_{k=1}^{K} Y_{k} V_{k} \frac{d h_{k}}{d x}+\sum_{k=1}^{K} W_{k} h_{k} \dot{\omega}_{k}=0
$$

where $\lambda$ is the thermal conductivity, $h$ is the mixture specific enthalpy, $h_{k}$ is the species specific enthalpy, $W_{k}$ are the species molecular weights and $\dot{\omega}_{k}$ is the rate of consumption. Both $h$ and $h_{k}$ are computed from fundamental thermodynamic theories as the summation of the ideal-gas enthalpy plus a departure function that accounts for dense fluid effects (see Section 3). It includes both the heat of formation and the sensible enthalpy. Heat losses due to radiation are neglected. Below, we confirm their minor importance compared to the energy conversion rate and heat conduction rate. (See section 5 ) For ideal gases, Eq. 5 reduces to

$$
2 F c_{p} \frac{d T}{d x}-\frac{d}{d x}\left(\lambda \frac{d T}{d x}\right)+\rho \frac{d T}{d x} \sum_{k=1}^{K} c_{p_{k}} Y_{k} V_{k}+\sum_{k=1}^{K} W_{k} h_{k} \dot{\omega}_{k}=0
$$

where $c_{p}$ is the specific heat at constant pressure. The viscosity $\mu$ and thermal conductivity $\lambda$ are to be evaluated both with ideal gases and with empirically correlated functions that were developed to extend kinetic gas theory to include dense fluids [35].

$V_{k}$ is the diffusion velocity and it is evaluated using the multicomponent formulation [16, in which pressure effect has been neglected.

$$
V_{k}=\frac{1}{X_{k} \bar{W}} \sum_{j=1}^{K} \mathcal{D}_{k j} W_{j} \frac{d X_{j}}{d x}-\frac{\mathcal{D}_{k}^{T}}{\rho Y_{k}} \frac{1}{T} \frac{d T}{d x}
$$

$X_{k}$ are the species mole fractions, $\bar{W}$ is the mean molecular weight, $\mathcal{D}_{k j}$ are the ordinary multicomponent diffusion coefficients, and $\mathcal{D}_{k}^{T}$ are the thermal-diffusion coefficients. Ideal gas thermodynamic and transport 
properties, as well as the reaction rates are obtained from the CHEMKIN 36] library of subroutines. The second term on the right hand side of Equation 7, also known as Soret effect, is in general neglected. However, for comparison purposes, we perform a check including this effect for one of our cases at 100 atm (see the discussion in Section 6.3 and Figure 11).

A simplified version of Eq. 5 results from the approximation that the enthalpy of the mixture $h=\sum_{k} Y_{k} h_{k}$ in the convective term.

$$
2 F \sum_{k=1}^{K} Y_{k} \frac{d h_{k}}{d x}-\frac{d}{d x}\left(\lambda \frac{d T}{d x}\right)+\rho \sum_{k=1}^{K} Y_{k} V_{k} \frac{d h_{k}}{d x}+\sum_{k=1}^{K} W_{k} h_{k} \dot{\omega}_{k}=0
$$

Eq. 8has been used in the literature as a real-gas model [10, 18, 20, 21]. However, the underlying assumption is that $h=\sum_{k} Y_{k} h_{k}$, which is not necessarily satisfied when dealing with real gases.

The three forms of the energy equation (Eq. 5. Eq. 6, and Eq. 8) are employed in this study for comparison purposes.

The eigenvalue $H=$ constant, the function $G=d F / d x$, Eq. 3 and 4 together with the energy equation and the equation of state (see Section 3), form a well-posed boundary-value problem, where the unknowns are $H, F, G, Y_{k}, T$ and $\rho$. For the given chemical mechanism involving $K$ species, the total number of differential equations is $K+3$ and the number of difference equations is $N(K+3)$, where $N$ is the number of grid points. Plug-flow boundary conditions are specified at the exit of the nozzles. As described in [37], these conditions can be reproduced accurately in laboratory experiments. In the following, $F$ and $O$ stand for fuel and oxidizer, respectively. At $x=0$ :

$$
F=\frac{\rho_{F} u_{F}}{2}, \quad G=0, \quad T=T_{F}, \quad Y_{k}=\left(Y_{k}\right)_{F}
$$

At $x=L$ :

$$
F=\frac{\rho_{O} u_{O}}{2}=-\frac{\rho_{F} u_{F}}{2}, \quad G=0, \quad T=T_{O}, \quad Y_{k}=\left(Y_{k}\right)_{O}
$$

We prescribe $u_{F}=1 \mathrm{~m} / \mathrm{s}$. The mass flux at $x=L$ is set to be equal in magnitude to the mass flux at $x=0$ with opposite sign. Thus, $u_{O}$ is also prescribed. Previous articles such as [20, 21] match the momentum

luxes instead of mass fluxes. The choice is arbitrary and the consequences are mild as shown in Appendix B. In our study, the Reynolds Number in the vicinity of the stagnation plane layer is, at most, of the order of $10^{4}$. Later, we explain how turbulence can be avoided. (See section 5.)

With regard to chemical kinetics, the detailed reaction mechanism GRI 3.0 [38] is selected, which consists of $K=53$ species and 325 elementary reactions. Available reaction mechanisms were developed at relatively low pressures. These mechanisms bring the species into chemical equilibrium, which in the classical form is based on partial pressures. At elevated pressures, however, chemical equilibrium is based on fugacities. We perform a check in Appendix A and validate that the differences are small. An unresolved concern arises with the use of existing chemical kinetic mechanisms (pathways) at very high pressures, since there is a lack of experimental validation.

\section{Equation of State}

The Soave-Redlich-Kwong Equation of State (EoS) is selected because of its reasonable accuracy for a wide range of fluid states [39].

$$
p=\frac{R_{u} T}{v-b}-\frac{a}{v(v+b)}
$$

This empirical equation, which may be rearranged to a cubic form with regards to the molar volume $v$, has two parameters $a$ and $b$, which are constants for single-component fluids, but become composition, pressure, and temperature dependent in the multicomponent version. The cubic form in terms of the compressibility factor $Z$ is

$$
Z^{3}-Z^{2}+\left(A-B-B^{2}\right) Z-A B=0
$$


where

$$
Z \equiv \frac{p v}{R_{u} T} \quad ; \quad A \equiv \frac{a p}{\left(R_{u} T\right)^{2}} \quad ; \quad B \equiv \frac{b p}{R_{u} T} .
$$

The parameter mixing rules of the Soave-Redlich-Kwong EoS are employed [40:

$$
a=\sum_{i=1}^{K} \sum_{j=1}^{K} X_{i} X_{j}\left(a_{i} a_{j}\right)^{0.5}\left(1-k_{i j}\right) \quad ; \quad b=\sum_{i=1}^{K} X_{i} b_{i}
$$

The pure species attractive and repulsive parameters $a_{i}$ and $b_{i}$ may be obtained from the species critical points as

$$
\begin{aligned}
& a_{i}=a_{c i} \alpha_{i} ; a_{c i}=0.42748 \frac{\left(R_{u} T_{c i}\right)^{2}}{P_{c i}} ; \alpha_{i}^{0.5}=1+S_{i}\left(1-T_{r i}^{0.5}\right) \\
& S_{i}=0.48508+1.5517 \omega_{i}-0.15613 \omega_{i}^{2} ; b_{i}=0.08664 \frac{R_{u} T_{c i}}{P_{c i}}
\end{aligned}
$$

where $T_{c i}$ and $P_{c i}$ are the critical temperature and critical pressure of mixture component $i, k_{i j}$ is the characteristic binary interaction constant and $\omega_{i}$ are the acentric factors. These values are taken from the literature 41. Sub-index $r$ stands for "reduced" and equals the property temperature or pressure divided by its critical value. All the attractive and repulsive parameters of chemically stable species such as $\mathrm{H}_{2}, \mathrm{O}_{2}, \mathrm{~N}_{2}, \mathrm{H}_{2} \mathrm{O}$, or metastable species like $\mathrm{H}_{2} \mathrm{O}_{2}$, may be determined from critical states conditions. However, chemically unstable species such as radicals do not have associated critical states, thus not allowing straightforward calculation of their attractive and repulsive terms. Assuming that the $i$ th species is a Lennard-Jones gas, for instance, it is possible to estimate the critical volume $v_{c, i}$ and the critical temperature $T_{c, i}[42$ and to obtain that

$$
a_{i}=(5.55 \pm 0.12) n \epsilon_{i} \sigma_{i}^{3} ; \quad b_{i}=(0.855 \pm 0.018) n \sigma_{i}^{3}
$$

where $n$ is the Avogadro number, $\sigma_{i}$ and $\epsilon_{i}$ are the molecular diameter and Lennard-Jones potential well depth of the $i$ th species, respectively.

The specific enthalpy departure function is given by

$$
h-h^{*}=\frac{1}{\bar{W}}\left\{R_{u} T(Z-1)+\int_{\infty}^{v}\left[T\left(\frac{d P}{d T}\right)_{v}-P\right] d v\right\},
$$

where $h^{*}$ is the enthalpy for an ideal gas at the given temperature and with the same composition. This departure function can be determined using the EoS [43]:

$$
h-h^{*}=\frac{1}{\bar{W}}\left[R_{u} T(Z-1)+\frac{T \frac{d a}{d T}-a}{b} \ln \frac{Z+B}{Z}\right]
$$

The species specific enthalpy $h_{k}$ is also evaluated using Equation 18, where the mixture parameters are replaced by those corresponding to the individual species.

\section{Solution Method}

A new simulator program has been written, which contains the mathematical model and boundary conditions. The differential equations are discretized using conventional finite-differencing techniques for nonuniform mesh spacing. Central differences are used for diffusive terms, with truncation error that is second-order. For better convergence, convective terms are discretized with upwind differencing, using the sign of the velocity to choose in which direction the spatial difference should go. For such terms, the 
truncation error is of first-order, leading to what is often called "artificial diffusion", but this form avoids unwanted oscillations during the solution on a coarse mesh. The simulator program calls the boundaryvalue problem solver TWOPNT 44 to determine the steady-state solution. In the counterflow-diffusionflame problem, the cost of forming and factoring the Jacobian matrix can be very expensive. For this reason, TWOPNT is based on a modified Newton's iteration method in which the Jacobian matrix is retained through several steps and re-evaluated only periodically. The iteration process begins from a solution guess with a coarse mesh. When a steady-state solution is found for a particular mesh, TWOPNT constructs a succession of finer grids with more points located where the solution changes abruptly until mesh independence is achieved. The criterion that determines grid independence (defined in [44]) is based on two tolerance values chosen by the user. We used 0.5 for both. If the steady-state search fails, TWOPNT undertakes a transient evolution solely to replace the starting guess by a better estimate. The evolution here is a pseudo-transient process, valuable only for producing the steady asymptote. The following timedependent terms are added to the left side of the momentum, energy and species equations, respectively:

$$
\text { Momentum: } \frac{d G}{d t}, \quad \text { Species: } \rho \frac{d Y_{k}}{d t}, \quad \text { Energy: } \rho \frac{d h}{d t}-\sum_{K} \rho h_{k} \frac{d Y_{k}}{d t} \text {. }
$$

The transient term used with the simplified forms of the energy equation (Eq. 6. and Eq. Eq. 80 is $\rho c_{p} \frac{d T}{d t}$.

Note that the true transient equations would have a time derivative in the continuity equation, thereby disallowing the creation of a stream function until a steady-state is reached. However, these pseudo-transient equations still give asymptotically the steady state while allowing a stream function to be created during the transient period.

Simulations at higher pressures can present convergence difficulties. So, when the pressure is increased, the solution of a previous case at a lower pressure is employed as the initial guess.

\section{Verification and Validation}

In order to validate our code, temperature profiles have been compared against experimental data at the highest possible pressure values. Temperature profiles are taken from [23] at $0.2,0.4$ and $0.8 \mathrm{MPa}$. We ran simulations with the same boundary conditions and domain size, entailing our most complete set of high-pressure corrections. As shown in Figure 2, there is good agreement between our computations and the experimental results.

Validation at $100 \mathrm{~atm}$ is also presented against numerical results from [21] for a methane-oxygen system where the strain rate is $1000 \mathrm{1} / \mathrm{s}$. Figure $2 \mathrm{~b}$ highlights a small difference in peak temperature. The source of this deviation could be due to the use of different chemical kinetics schemes or the different sub-models. To answer this question, we obtained a solution with our model and a reduced chemical kinetic mechanism that entails 19-species and 15 steps 45 . The curves belonging to our detailed and reduced chemistry sets are practically superimposed. The peak temperature resulting from the use of reduced chemistry is only 4 $\mathrm{K}$ hotter than for detailed chemistry, while the peak temperature in 21 is $60 \mathrm{~K}$ hotter than our detailed kinetics result. Thus, the deviation from 21] cannot be explained by the use of different chemical kinetic schemes and is primarily diffusion controlled. Consequently, our additional correction makes a modest difference at the given strain rate.

As mentioned in the Model section, a check on the importance of radiative effects was performed. To this effect, we estimated the radiative heat rate and compared it with the energy conversion rate and the conductive heat rate. The calculations on radiation are based on a method published elsewhere [46]. The radiative heat loss is computed as $q_{r a d}=\sum_{k=1}^{K} p_{k} \kappa_{k} \sigma\left(T^{4}-T_{b}^{4}\right)$, where $p_{k}$ is the partial pressure, $\kappa_{k}$ is the Planck mean absorption coefficient for species $k, \sigma$ is the Stefan-Boltzmann constant, and $T_{b}$ represents the temperature of the background environment $(300 \mathrm{~K})$. The Planck mean absorption coefficients depend on the species and temperature. They are taken from 5th degree Gaussian curve fittings reported in [46]. Four 


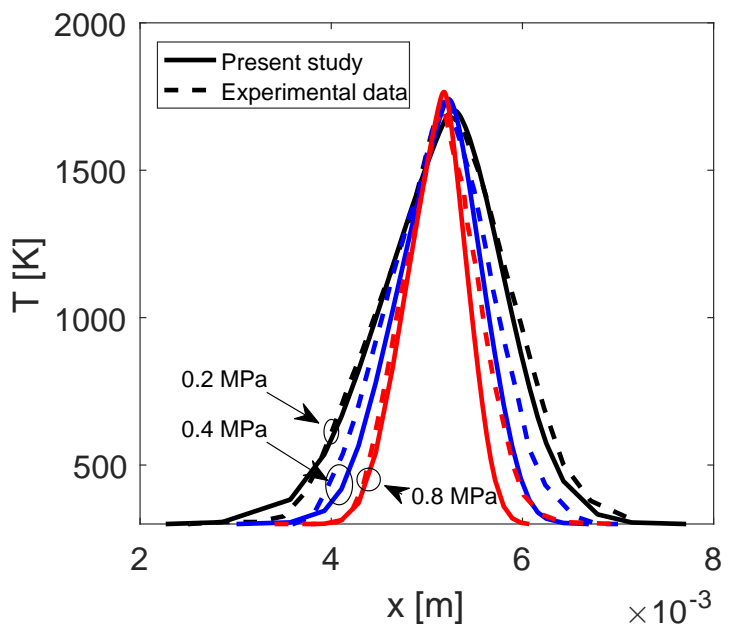

(a)

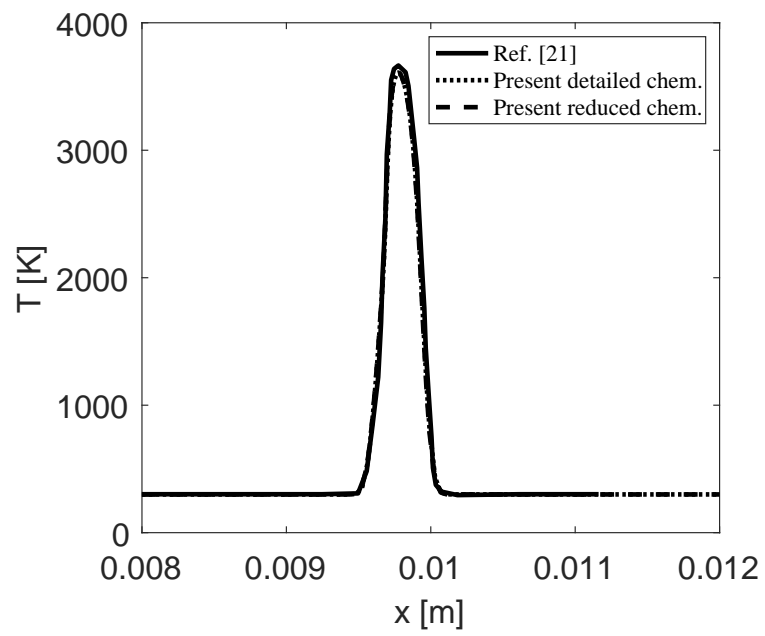

(b)

Figure 2: (a) Temperature profile benchmarking with [23; (b) Benchmarking with 21] for a $\mathrm{CH}_{4}-\mathrm{O}_{2}$ system at 100 atm.

species $\left(\mathrm{CO}_{2}, \mathrm{H}_{2} \mathrm{O}, \mathrm{CO}, \mathrm{NO}\right)$ are used. The results are illustrated in Figure 3, which shows a blow up of the heating zone from Case 6 (real-gas model without water, see section 6) at a pressure of $100 \mathrm{~atm}$.

Figure 3 a shows temperature and three black curves that represent conductive heat rate (solid), radiative heat rate (dotted), and energy conversion rate (dashed). The energy conversion rate and heat rate by conduction are practically matched. The radiation curve is on the zero line. Figure $3 \mathrm{p}$ highlights the small magnitude of the radiative rate compared to conduction and conversion. Sub-figures 3 ; and $3 \mathrm{~d}$ display the same quantities for Case 8c (real-gas model with the highest studied water content, see section 6), also at a pressure of $100 \mathrm{~atm}$. The radiative heat rate is now greater than it was for Case 6 , but its value is still two orders of magnitude below the other two energy rates. Thus, we conclude that radiation can be neglected.

Let us discuss how the flow can remain laminar even at the high Reynolds number at high pressures. We identify four potential sources of turbulence: (i) turbulence coming from the interior of the two burner ducts; (ii) buoyancy instability; (iii) turbulence being generated in the shear layer of the gas jet; and (iv) turbulence being generated in the shear layer near the stagnation plane region. See the sketch presented in Figure 4 a where these four regions have been highlighted. Below we review each one of these turbulence 


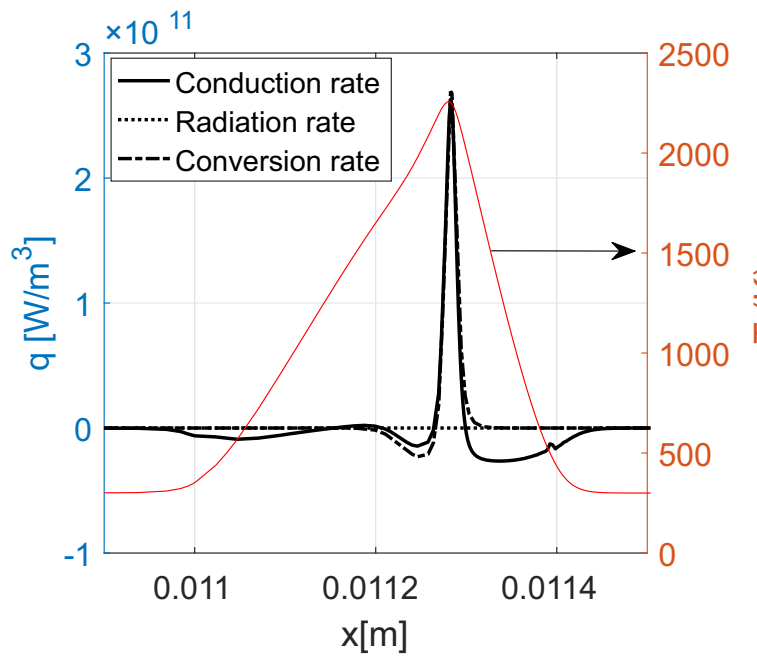

(a)

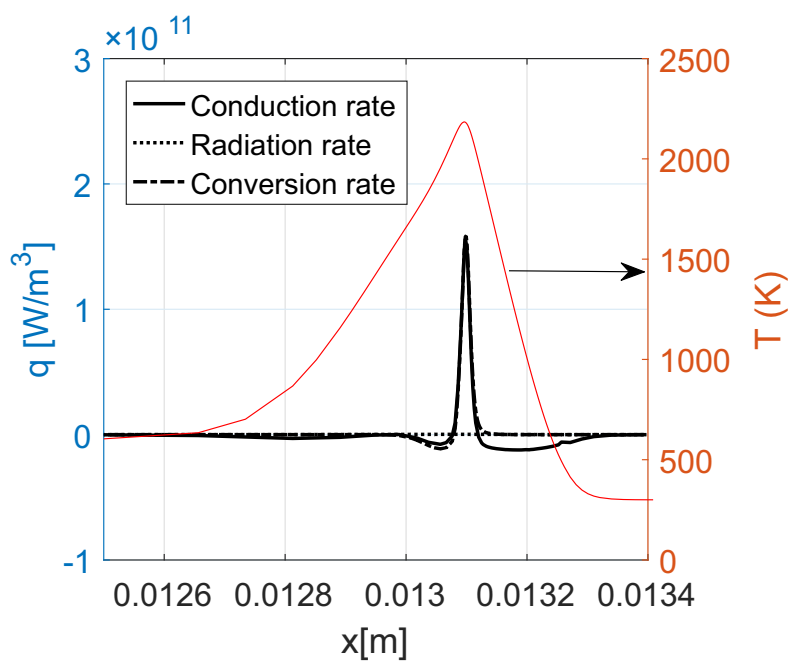

(c)

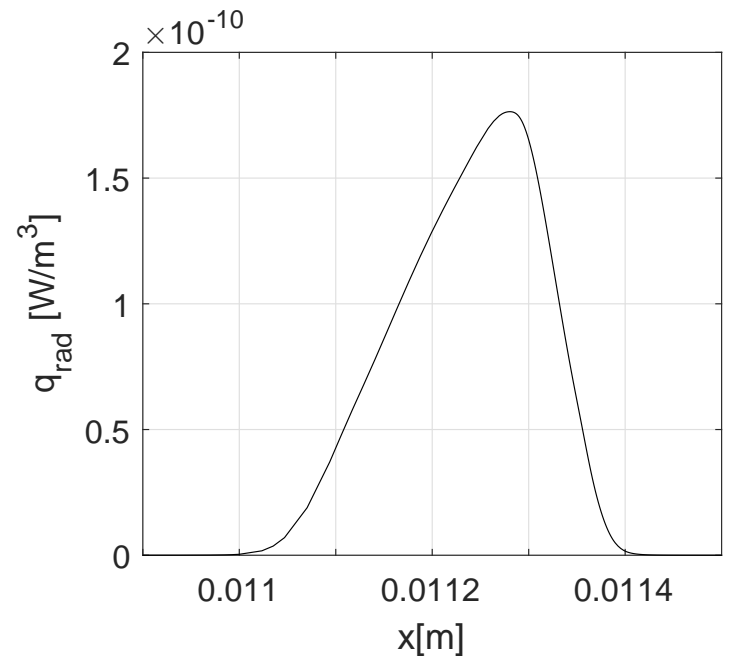

(b)

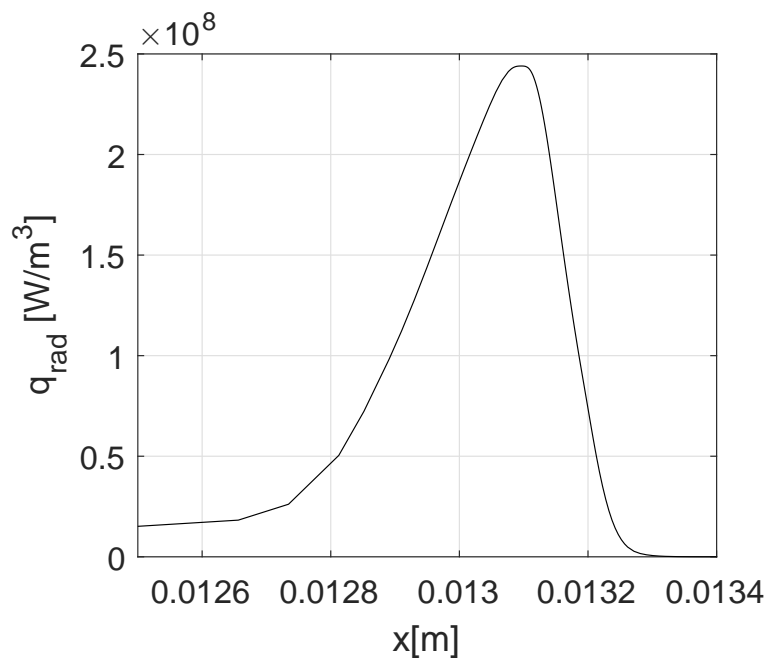

(d)

Figure 3: Comparison of radiation, conduction, and energy conversion rates at 100 atm for Case 6 in Sub-figures a and b, and Case 8c in Subfigures c and d. 


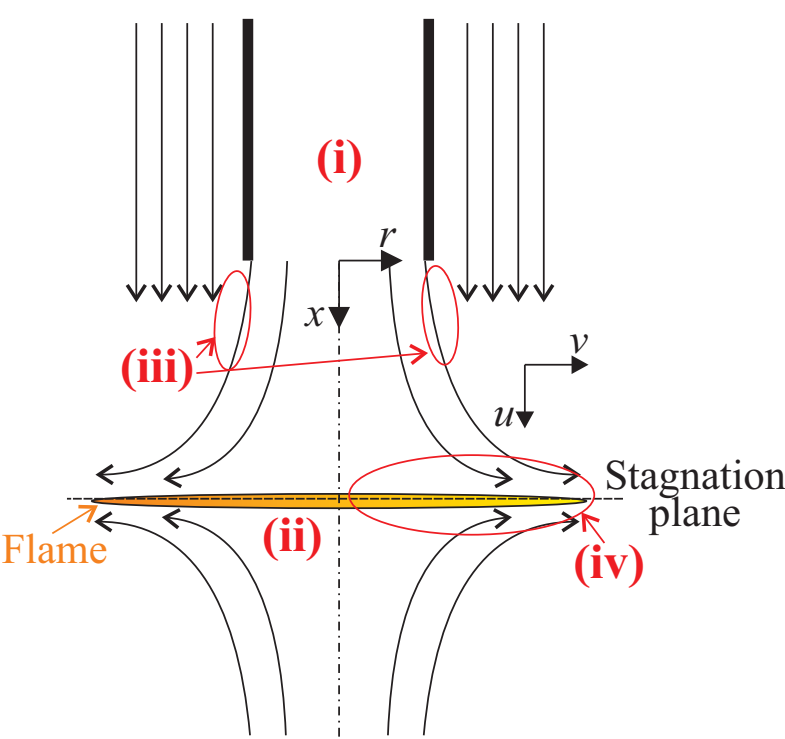

(a)

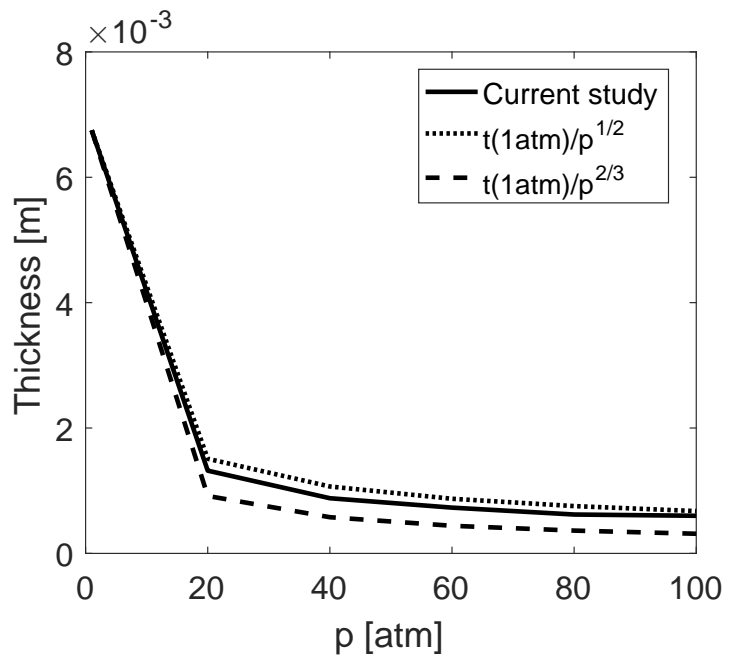

(b)

Figure 4: (a) Turbulence sources; (b) Thickness of the layer with density variation vs. T.

(i) We can assume that the nozzles are equipped with suitable porous materials or very fine grids such that any eddies entering from the nozzles would decay in a very short distance after entering the studied domain.

(ii) A discussion on the appearance of instabilities due to buoyancy is presented in [37. The authors report that special care is needed to produce turbulence when it is needed. They also note that laminar flow has been obtained at $25 \mathrm{~atm}$ and laminar behavior at higher pressures can be expected if the layer with density variation has its thickness decreasing as $p^{-2 / 3}$ or faster. See Figure $4 \mathrm{~b}$, which shows the variation of flame thickness for Case 6 in this study (solid), the variation with $p^{-1 / 2}$ (dotted, from the literature), and the variation with $p^{-2 / 3}$ (dashed). There is no significant difference between the three curves in terms of order of magnitude. Hence, we can also assume very little instability will appear from source (ii).

(iii) Turbulence generated in the outer shear layer of the jet may be avoided with the use of a co-flow, which in fact is a common practice in actual experiments.

(iv) To justify our neglect of turbulence from this fourth source, we include an order of magnitude analysis using a well-established equation for the calculation of the advective flux, diffusion, generation, and dissipation of turbulent kinetic energy in a shear-layer. The equation is taken from [47:

$$
\rho \frac{D k}{D t}=-\frac{\partial}{\partial y}\left(\rho \overline{v^{\prime} k}+\overline{v^{\prime} p^{\prime}}\right)-\rho \overline{u^{\prime} v^{\prime}} \frac{\partial u}{\partial y}-\sum\left(\frac{\partial \overline{u_{i}^{\prime}}}{\partial x_{j}}\right)^{2}
$$

The three terms on the right hand side represent diffusion, production, and dissipation, respectively. We identified that the production term is dominant with respect to dissipation. So, we look at the worst case scenario where only production plays a role. We have that $\left(\overline{u^{\prime} v^{\prime}}\right)=\mathcal{O}(k)$ which arbitrarily maximizes production rate by assuming that both velocity components have comparable magnitudes and the fluctuation velocity components are well correlated. $\partial u / \partial y$ in our coordinate system is $\partial v / \partial x$. We obtain the order of magnitude of this quantity from the solution of Case 6 at $100 \mathrm{~atm}$. Taking the order of magnitude of the radial direction as $\mathcal{O}(r)=10^{-2} \mathrm{~m}$, we get from Figure 5 that $\mathcal{O}(\partial v / \partial x)=10^{5} \mathcal{O}(r)=10^{3} 1 / \mathrm{s}$.

Therefore, a rough but conservative estimate neglecting any decrease in $k$ due to diffusion or dissipation yields

$$
\frac{1}{k} \frac{\Delta k}{\Delta t}=\mathcal{O}\left(10^{3} \frac{1}{\mathrm{~s}}\right)
$$




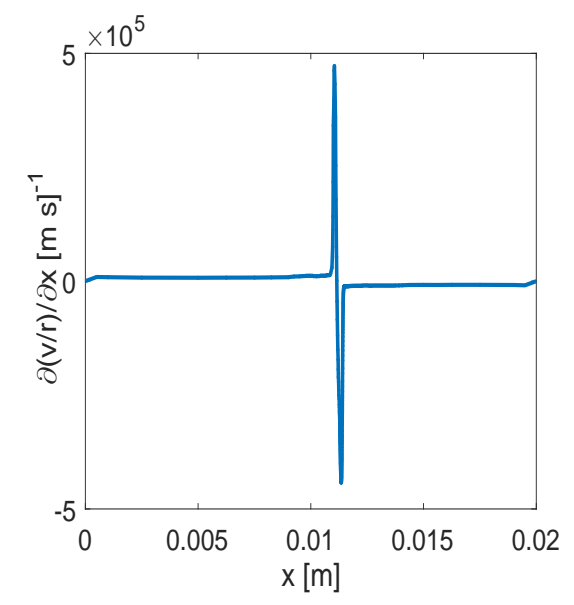

Figure 5: Derivative in the axial direction of $v / r$.

\section{Results}

Eight cases are studied as defined in Table 1 , each with varied pressure from 1 to $100 \mathrm{~atm}$. The distance between the two nozzles is $L=2 \mathrm{~cm}$, similar to what is used in typical experiments. Pure methane flows against air in Cases 1 through 7, where the temperature at both boundaries is $300 \mathrm{~K}$, and the fuel inflow velocity equals to $1 \mathrm{~m} / \mathrm{s}$. The air nozzle flow speed is calculated according to the $F$ boundary condition (see Equation 10). As mentioned in the Introduction section, one of our goals is to determine the relative importance associated with high-pressure rectifications. Differences across Cases 1-7 reflect three real-gas corrections: (I) replacement of the ideal-gas law by the cubic equation of state; (II) use of the real-gas energy equation (Eq. 5); and (III) modification of the viscosity and thermal conductivity.

No corrections are used in Case 1. Only the transport properties are corrected in Case 2. Case 3 is equipped with the cubic EoS and no other corrections. Case 4 entails the real-gas energy equation (Eq. 5), ideal-gas law, and no transport modification. A combination of corrections (I) and (II) are used in Case 5, 
Table 1: Studied Cases

\begin{tabular}{|c|c|c|c|}
\hline Case & Correction I: EoS & Correction II: Energy equation & Correction III: $\mu$ and $\lambda$ \\
\hline 1 & Ideal & Ideal (Eq. 6) & Ideal \\
\hline 2 & Ideal & Ideal (Eq. 6 ) & Corrected \\
\hline 3 & Cubic & Ideal (Eq. 6) & Ideal \\
\hline 4 & Ideal & Corrected (Eq. 5 & Ideal \\
\hline 5 & Cubic & Corrected (Eq. 5 ) & Ideal \\
\hline 6 & Cubic & Corrected (Eq. 5 ) & Corrected \\
\hline 7 & Cubic & As in literature (Eq. 8 & Corrected \\
\hline $8 \mathrm{a}$ & Equivaler & to Case 6 - Fuel inlet contains 1 & $\%$ water vapor. \\
\hline $8 \mathrm{~b}$ & Equivaler & to Case 6 - Fuel inlet contains 2 & $\%$ water vapor. \\
\hline $8 \mathrm{c}$ & Equivaler & to Case 6 - Fuel inlet contains 4 & \% water vapor. \\
\hline
\end{tabular}

while Case 6 presents all three corrections. Case 7 is used to study the error introduced by assuming that the enthalpy of the mixture equals the summation of the enthalpy of each component times its mass fraction in the energy equation. Namely, it uses a more simplified version of the energy equation (Eq. 8 as presented in previous studies [10, 18, 20, 21] instead of the Eq. 5].

In addition, Case 8 is used to study the effect of water vapor content premixed with the methane in the fuel stream. All high-pressure corrections are enabled in this case. The temperature at the exit of the fuel stream is raised to $600 \mathrm{~K}$, a value that is above the saturation temperature of water at $100 \mathrm{~atm}$. See Table 1 for a summary of these cases.

The comparative study between cases 1-6 is shown in sub-section 6.1. The effects of the new enthalpy approach in the energy equation are discussed in sub-section 6.2. Results and discussion of the cases entailing the most complete physical model with and without premixed water vapor (Cases 6 and 8) are shown in sub-sections 6.3 and 6.4

\subsection{Comparing Cases 1-6}

To better assess the effects of the three corrections, solutions for Cases 1 through 6 at 100 atm are plotted together in Figures 6 and 7. For a given pressure, temperature, composition, and fuel-inlet velocity, the most accurate mass flow rates at the boundaries are given by the real-gas model. Use of simplified models such as the ideal-gas law, yield a less accurate density value at the boundaries which of course affects the mass flow rates. The choice here is arbitrary: (i) either keep the fuel-inlet velocity, temperature, pressure, and composition fixed at the boundaries and only change the model; or (ii), if we wanted to keep the mass flow rates the same when comparing between ideal and real models, we would have to modify the temperature, pressure, or composition such that the density at the boundaries matched between real and ideal models. (Or we must change the inlet velocity which would affect the strain rate.) We chose the former approach (i) because they present as the given parameters the more easily measurable quantities. As mentioned above, we prescribe the velocity at the exit of the fuel nozzle and match the mass flow rates of the two nozzles. Note that under the latter approach (ii), we would be studying two different problems.

Sub-figures 6a, b, and d show two groups of almost overlapped curves, one containing Cases 1, 2, 4 and the other including Cases 3,5 and 6. These figures show the whole domain. A narrower domain is shown in the rest of the figures to gain resolution. A closer look to the velocity profiles shows that the correction in transport properties has a very small effect on the solutions. In contrast, application of the cubic equation of state introduces a substantial difference, which moves the flame and stagnation coordinate closer to the center plane. Correction of the energy equation causes more change than transport correction, but is less significant than replacing the equation of state. Furthermore, it moves the stagnation plane away from the center of the domain.

Sub-figure 6 $6 \mathrm{~d}$ shows the function $G$, which is related to the radial velocity $v$. The curves exhibit an almost linear behavior for most of the domain except for a thin layer around the flame. We can see a 
substantial change in the slope of the linear part of the curves for the cases entailing the cubic equation of

Sub-figures 7e and 7f show the percentage difference between ideal and corrected transport properties for the cases in which they are mended. The difference is about $3 \%$ in average in the flame regions, while it becomes $20 \%$ or more in the cold regions. The absolute value of transport properties at cold temperatures up even though the absolute difference is not so vast. Thus, great differences with respect to Case 1 were not expected. 


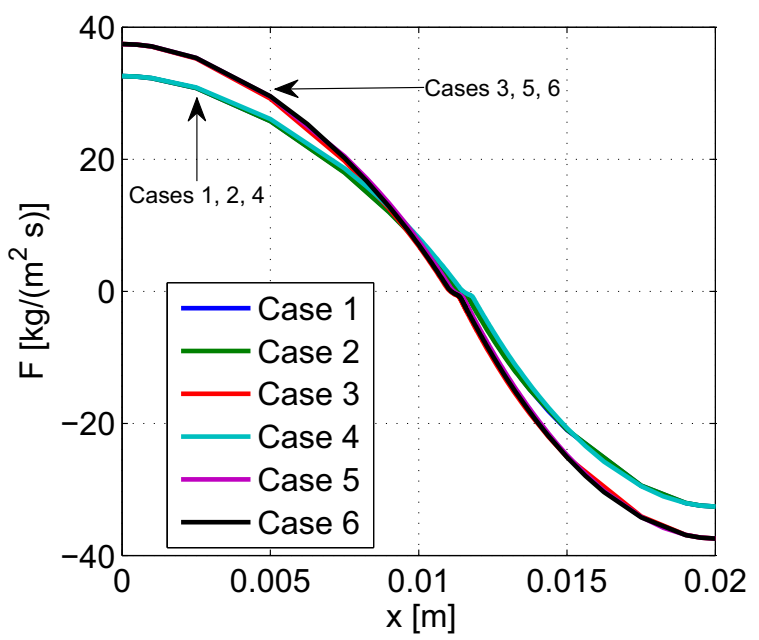

(a) $F$

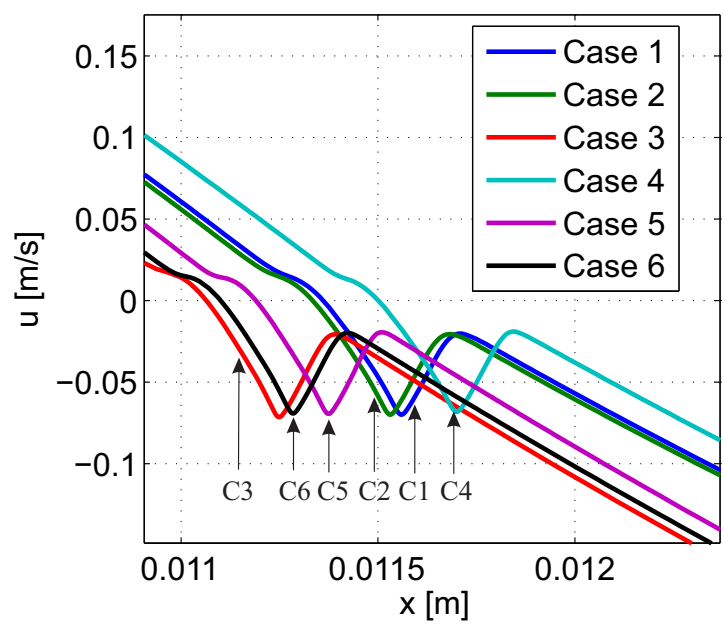

(c) Zoom of $u$

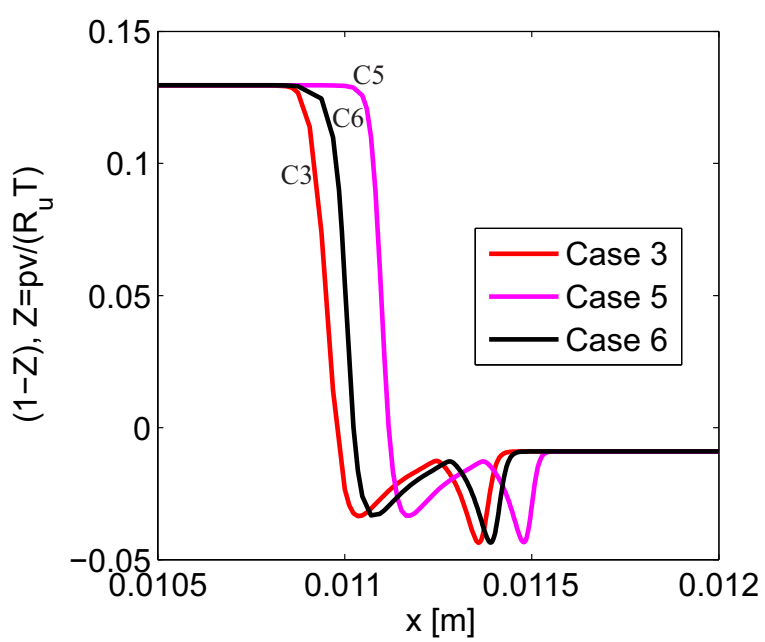

(e) $1-Z$

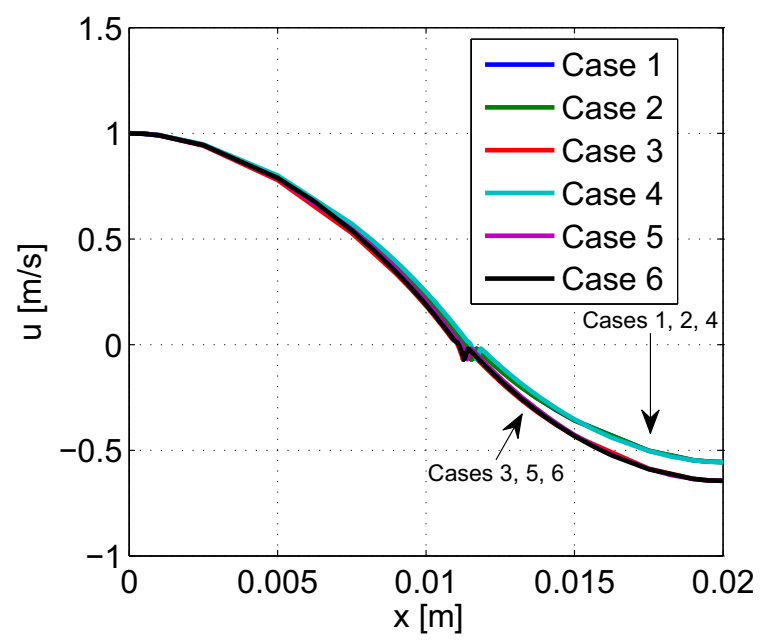

(b) $u$

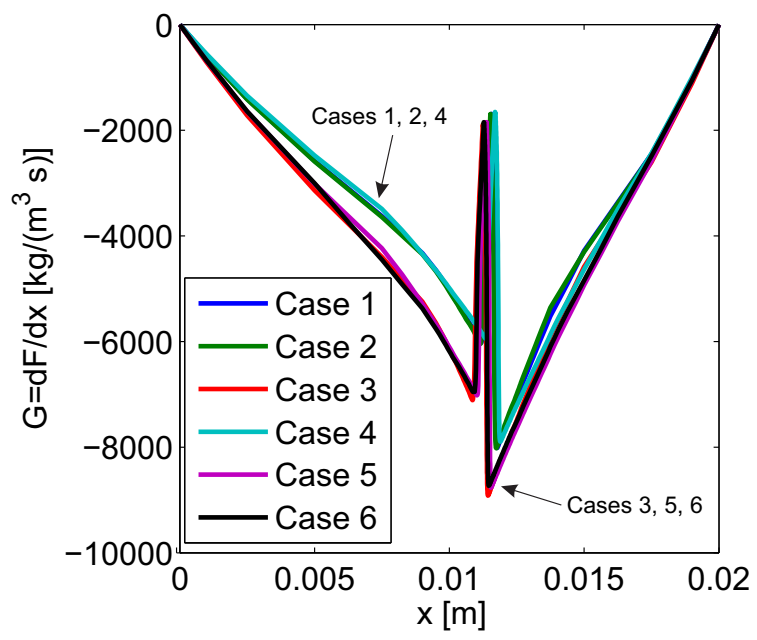

(d) $G$

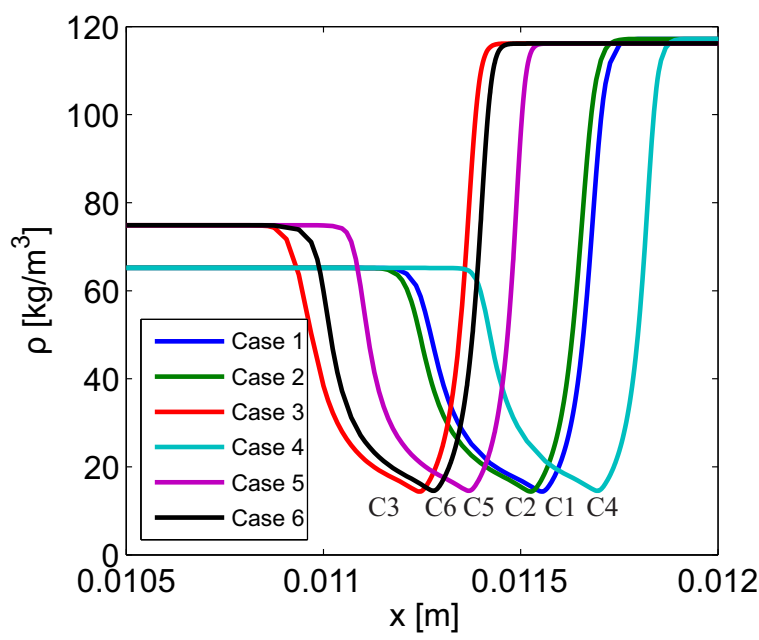

(f) $\rho$ 


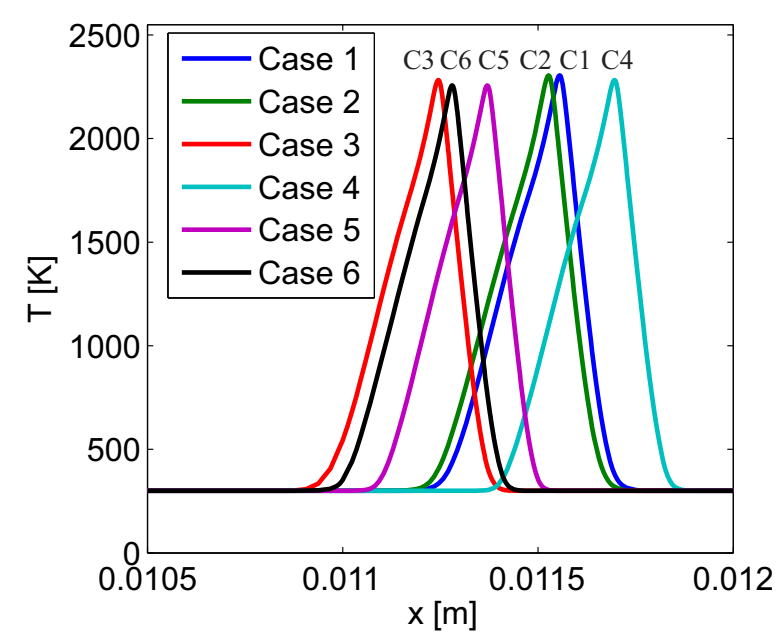

(a) $T$

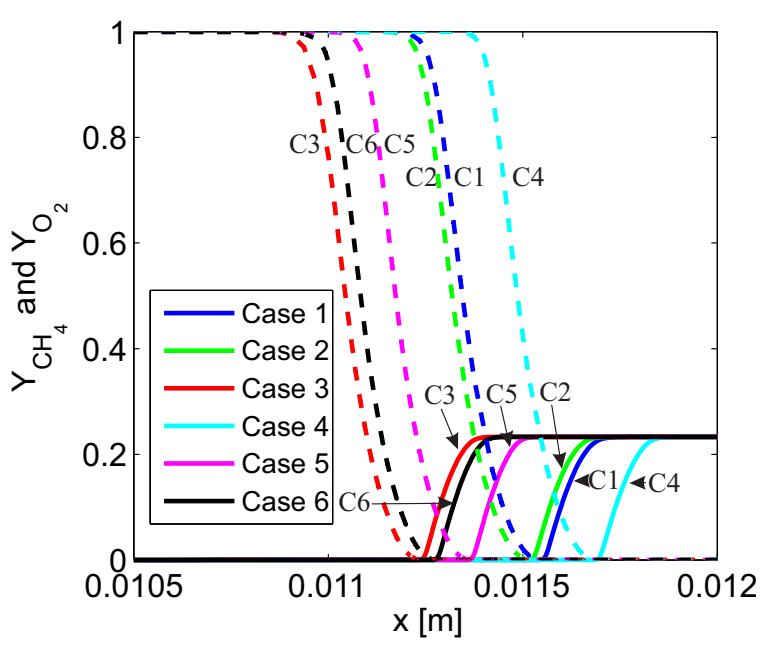

(c) Dashed: $Y_{\mathrm{CH}_{4}}$, Solid: $Y_{\mathrm{O}_{2}}$

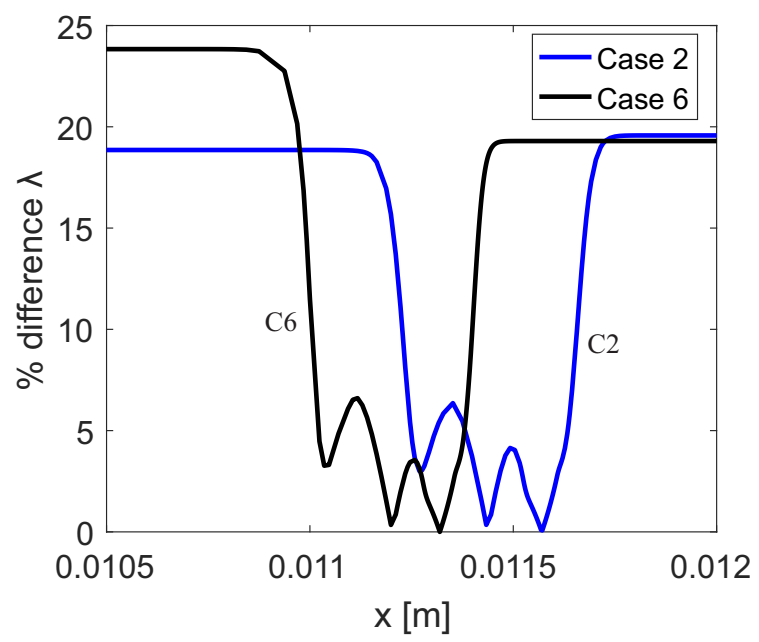

(e) $\% \lambda$ difference between ideal and corrected

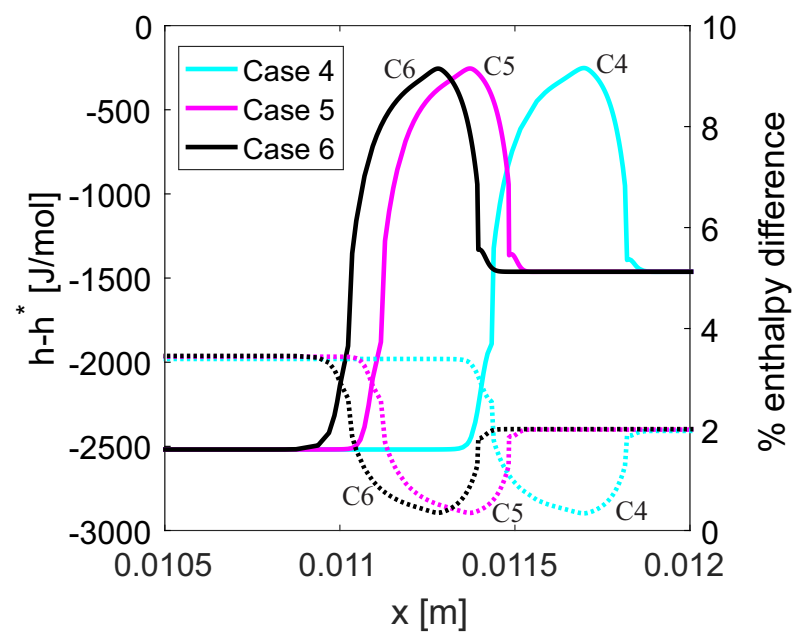

(b) Mixture enthalpy departure and $\%$ difference with sensible enthalpy

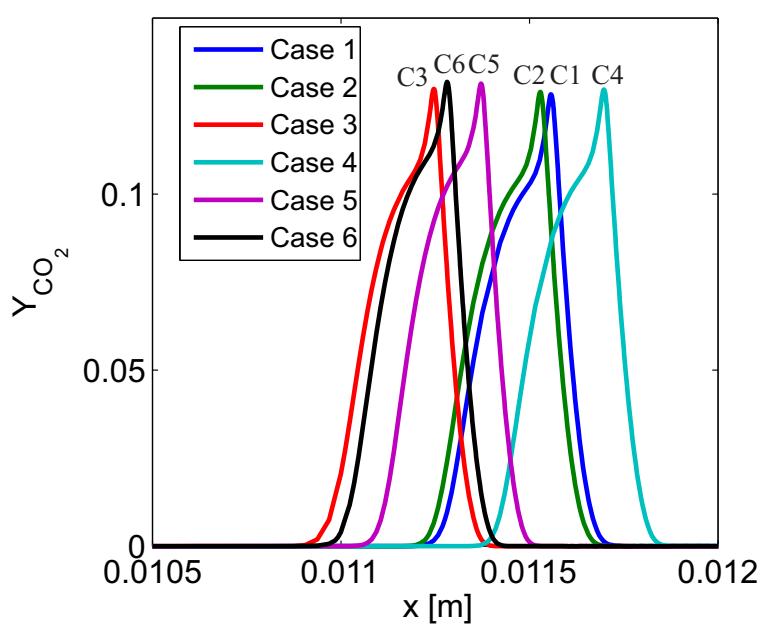

(d) $Y_{\mathrm{CO}_{2}}$

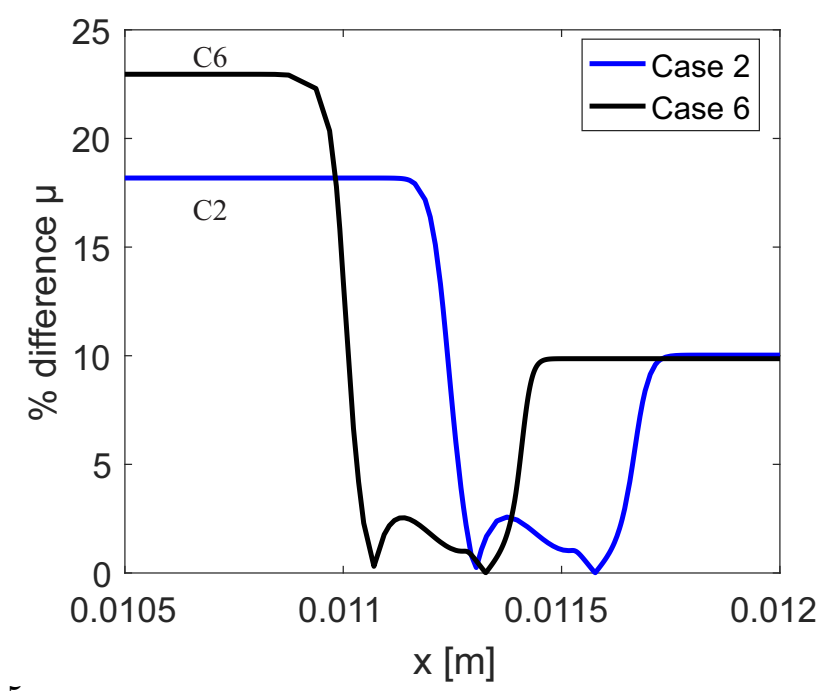

15

Figure 7: Comparison of variables between Cases 1-6 at $100 \mathrm{~atm}$. 


\subsection{New enthalpy approach: Case 6 vs. Case 7}

As discussed in Section 2, our most complete model differs from previous studies because we do not assume $h=\sum_{k} Y_{k} h_{k}$ in the convective term of the energy equation. To assess the consequences of such simplification, enthalpy and temperature profiles are contrasted between the two approaches in this section.

We study the behavior of the mixture enthalpy versus temperature at fixed composition in Figure 8 The composition is prescribed, taken from Case 7 at the $\mathrm{x}$-coordinate where the flame temperature peaks. Sub-figure 8 a portrays the enthalpy departure function of the mixture from Equation 17 (dotted lines) versus the same property but computed from the summation of the individual components times the mass fractions (dashed lines). The departure function is very close to 0 for both cases at atmospheric pressure, consistent with ideal-gas behavior. As pressure is increased, the departure becomes more important. At very high temperature, the departure is closer to zero than at cold temperatures, but it does not behave exactly as in the ideal situation. The enthalpy mixture calculation yields a positive departure above $700 \mathrm{~K}$, while the summation approach always yields negative values. The absolute values of differences between the mixture and the summation approaches are noticeable. Sub-figure $8 \mathrm{~b}$ shows the percentage difference between the mean enthalpy computed from the mixing rules and the mean enthalpy computed from the summation of components. The differences are bounded above by $1 \%$ below 10 atm. They become more important at $100 \mathrm{~atm}$, with differences reaching about $6 \%$ relative difference at the highest temperature. With this information, we would like to see what the effect of such differences is on the flame.

If we introduce the composition dependence that exists in the counterflow domain, for example for Case 7 , we see in Figure 9 a that the relative difference between computing the enthalpy directly from mixing rules or from the summation approach is below $2 \%$. With this information in mind, we now compare counterflow diffusion flame solutions between Cases 6 and 7 at various pressures. Sub-figure $9 \mathrm{~b}$ shows temperature percentage differences below $3 \%$, while flame location and structure are practically unaltered. 


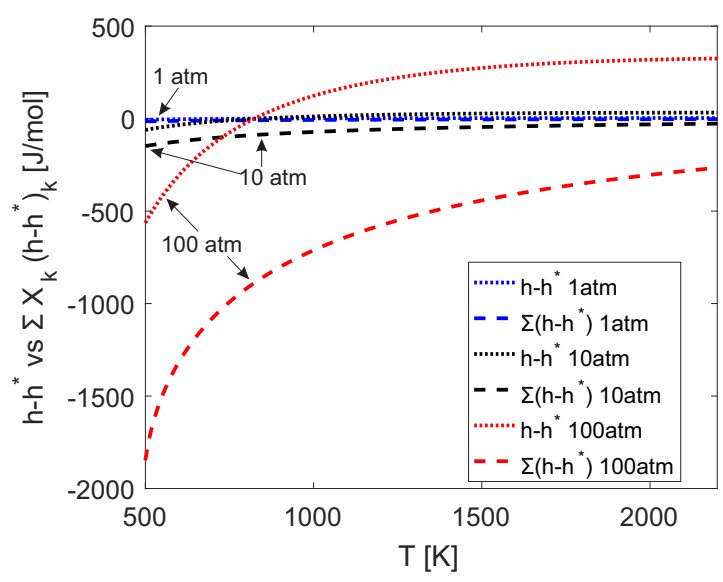

(a) $h-h^{*}$

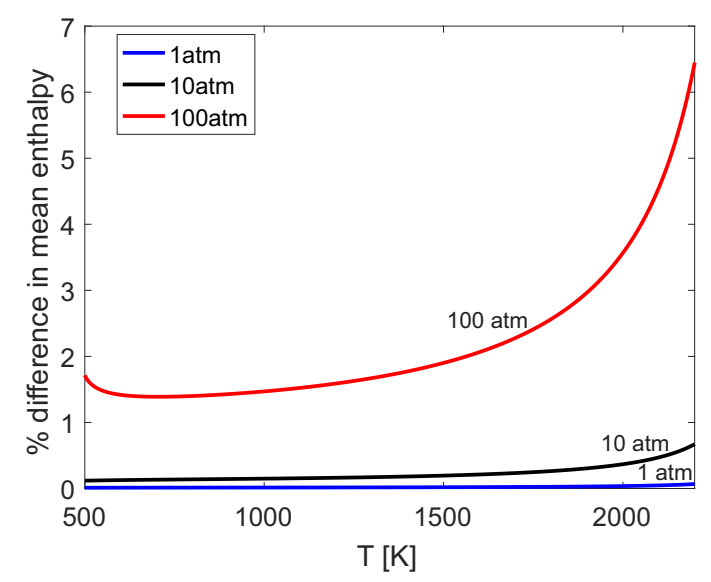

(b) $h$ relative difference between mixture and summation

Figure 8: Enthalpy curves (from Case 7 at flame peak location) versus $T$.

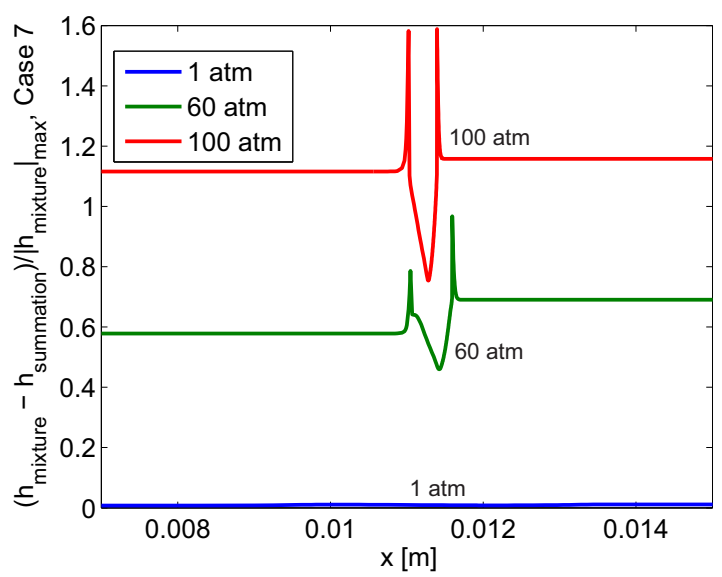

(a) Enthalpy relative difference

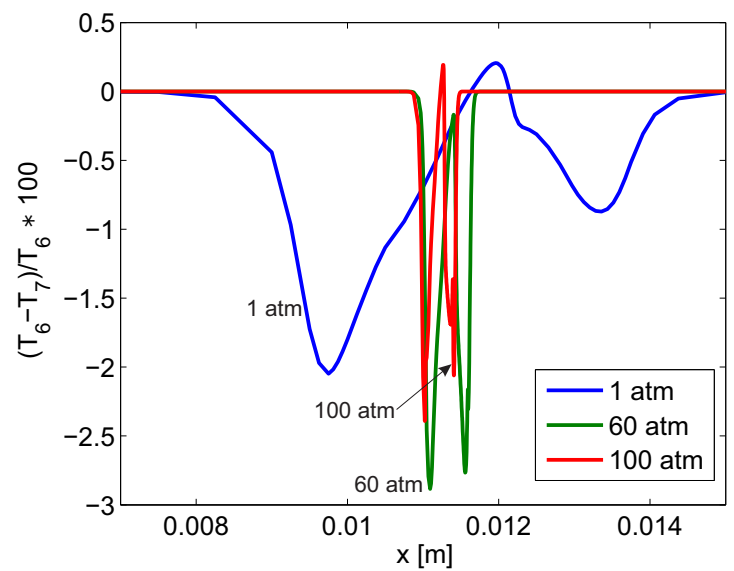

(b) $T$ relative difference between mixture and summation

Figure 9: Differences in enthalpy and temperature profiles between Case 6 and Case 7. 


\subsection{Case 6: Complete Model}

The goal of this section is to analyze Case 6 (which entails our most complete model) in order to obtain conclusions about the pressure effect on the flame and check if the flame structure complies with previously reported correlations with the pressure-weighted strain rate.

Solutions are presented in Figure 12. Sub-figure 12 shows velocity and temperature profiles. As expected from the momentum-flux ratio, the stagnation plane is to the right of the symmetry point $(x=L / 2)$. The stagnation plane would lie on the center of the domain if the momentum fluxes $\rho u^{2}$ at the two nozzles were balanced. However, given the fixed density values of methane and air at the nozzles, the boundary condition $F_{(x=L)}$ (see Eq. 10 implies that the momentum flux of the methane stream is greater than that of the air stream. Thus, stagnation occurs closer to the air nozzle. See Appendix B for a more detailed comparison between the two boundary conditions. The increase in pressure increases flame temperature and narrows flame width. The first increment in pressure causes the most substantial temperature rise, while further escalation in pressure does not imply such a high relative temperature increase. Higher pressure suppresses species dissociation which tends to bring the temperature down, and higher temperature favors dissociation. At higher pressures the kinetics are faster. So, the balance between the pressure and temperature effects on dissociation is determining. However, there is only so much energy that can be extracted from the reaction, so one can not expect a linear flame temperature increase with pressure, but an asymptotic behavior. The flame temperature at $1 \mathrm{~atm}$ equals $1,987 \mathrm{~K}$, while its value at $100 \mathrm{~atm}$ is $2,255 \mathrm{~K}$. The $\mathrm{x}$-coordinate where the maximum flame temperature occurs moves closer to the stagnation plane as pressure rises, but it always sits to its right, on the oxidizer side.

Previous publications such as 21] reported correlations between flame temperature and pressure $T_{m} \sim$ $p^{0.0474}$, and flame thickness with pressure-weighted strain rate $\delta \sim 1 / \sqrt{p a} . a$ is the strain rate defined as the maximum absolute velocity gradient in the flow field. The flame-thickness scaling obtained in this study was shown in Section 5 Figure 4 showing close agreement with the $\delta \sim 1 / \sqrt{p a}$ correlation. Another correlation for the heat-release rate was obtained in 20 . There, the authors present an analysis using average transport and thermodynamic properties in the flame zone and assume Lewis number of unity, yielding $\dot{q} \sim p^{0.534} \sqrt{a}$. As displayed in Figure 10a, our result shows that the heat-release rate correlates more accurately with the square root of $p$.

[48] provides experimental results for laminar counterflow diffusion flames at pressures below $3 \mathrm{MPa}$. A scaling for the flame temperature is identified, which we repeated in this paper for comparison purposes. The result is shown in Figure 10, where the normalized temperature and $x$-coordinate are $\hat{T}=\left(T-T_{o}\right) /\left(T_{M A X}-\right.$ $\left.T_{o}\right)$ and $\hat{x}=x / \delta_{d i f f}$, respectively. $\delta_{d i f f}=\sqrt{\frac{\alpha_{o}}{a} \frac{p_{o}}{p}}$, where $\alpha_{o}$ is a thermal diffusivity evaluated at a mean temperature of $1000 \mathrm{~K}$ and at $1 \mathrm{~atm}$ for the oxidizer stream, and $a$ is the strain rate. As the plot highlights, the curves at different pressures collapse to a single curve, as suggested in the provided reference. The curve at the highest pressure $(100 \mathrm{~atm})$ shows the greater departure, suggesting that this correlation might only be valid up to a certain pressure threshold. Real-gas transport properties might explain the departure at high pressures.

As anticipated in Section 5, a check has been performed on the effect of including the thermophoretic contribution to the diffusion velocity. At very high pressures, the flame thickness is very narrow and the temperature gradient is at its highest magnitude. Hence, one could argue that the Soret effect may play an important role. On the contrary, the result displayed in Figure 11 shows that the differences between including and neglecting the Soret effect are very small. Inclusion of this effect tends to increase the flame temperature slightly. The local velocity peak in the reaction zone also increases modestly.

Sub-figure 12. shows the mass fractions of methane and oxygen. The curves become much steeper at high pressures due to the smaller flame thickness. The following may be difficult to see by eye inspection and observations; they were made with a closer computational view. At $1 \mathrm{~atm}$, there is coexistence of both reactants near the region of maximum temperature, although mass fraction values are small (i.e. $<0.005)$. Methane that diffuses into the oxygen decays quickly, while oxygen diffuses a much longer distance into the methane side and past the stagnation plane. In contrast, at 100 almost no overlapping exists. For example, at $x=11.54 \mathrm{~mm}$, the mass fraction of both reactants is $<0.0005$ and it decays very quickly moving in either 


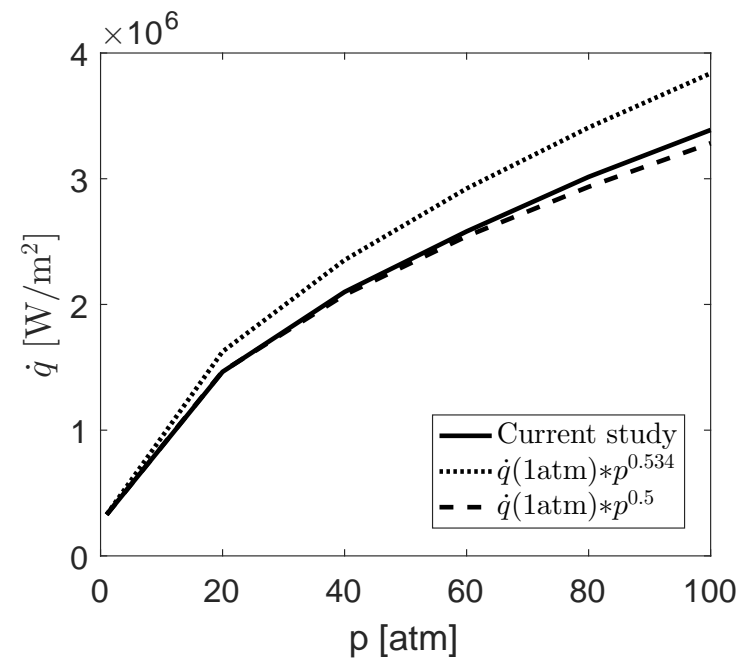

(a)

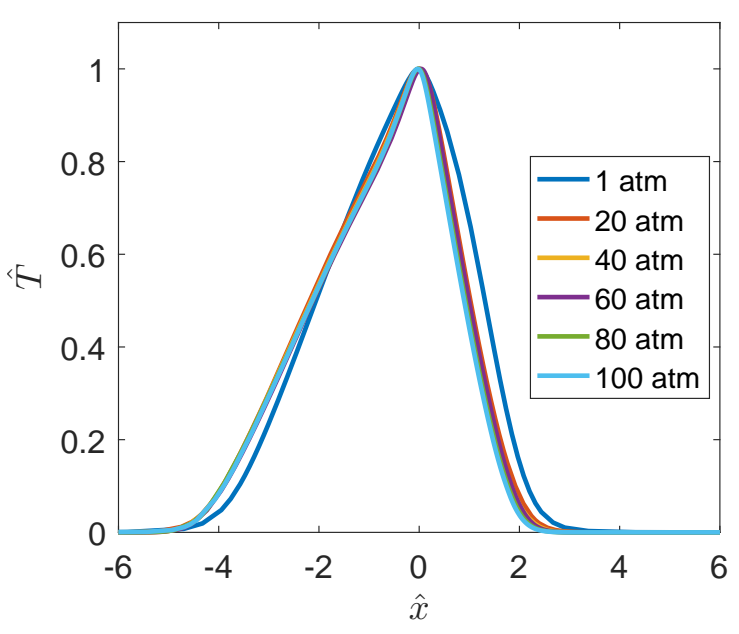

(b)

Figure 10: Heat-release rate and non-dimensional temperature scalings for Case 6 at $100 \mathrm{~atm}$.

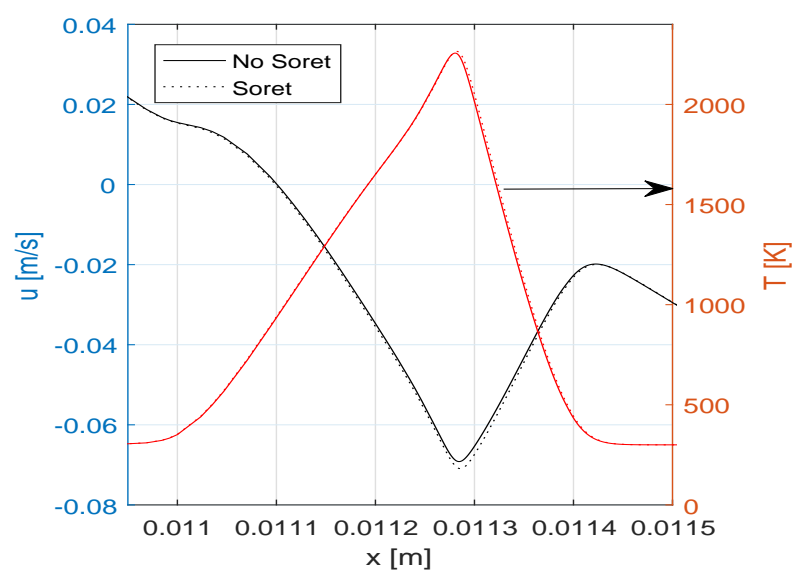

Figure 11: Soret effect on Case 6 at $100 \mathrm{~atm}$.

direction. Sub-figure 12 d represents carbon dioxide mass fractions. This combustion product is generated along a broader region at low pressure, and its presence narrows as pressure raises. $\mathrm{CO}_{2}$ mass fraction peak value also increases with pressure. Similarly to the flame temperature behavior, the most substantial increment in $\mathrm{CO}_{2}$ mass fraction occurs when pressure is raised from 1 to 20 atm. In comparison, further increments do not seem to yield such a strong effect. The reasoning behind this is the same as for the temperature profiles. 


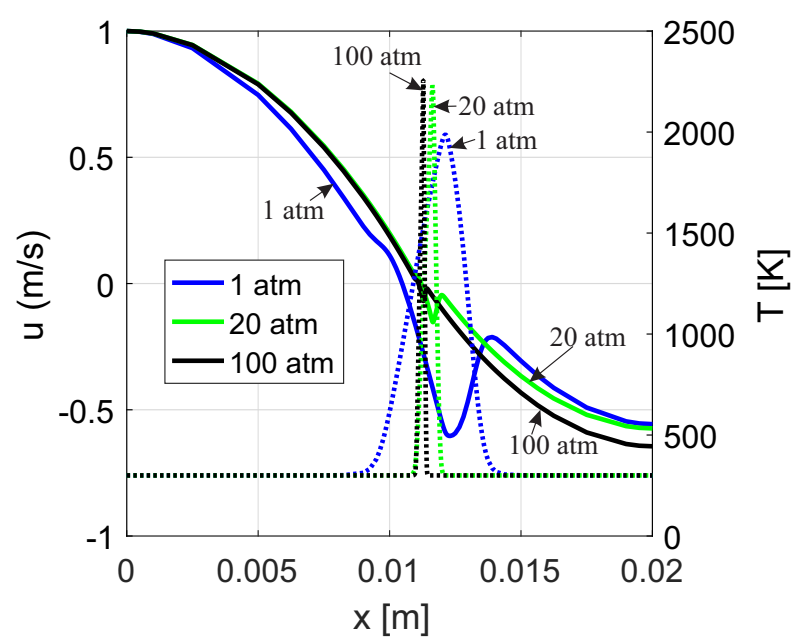

(a) Solid: $u$, Dotted: $T$

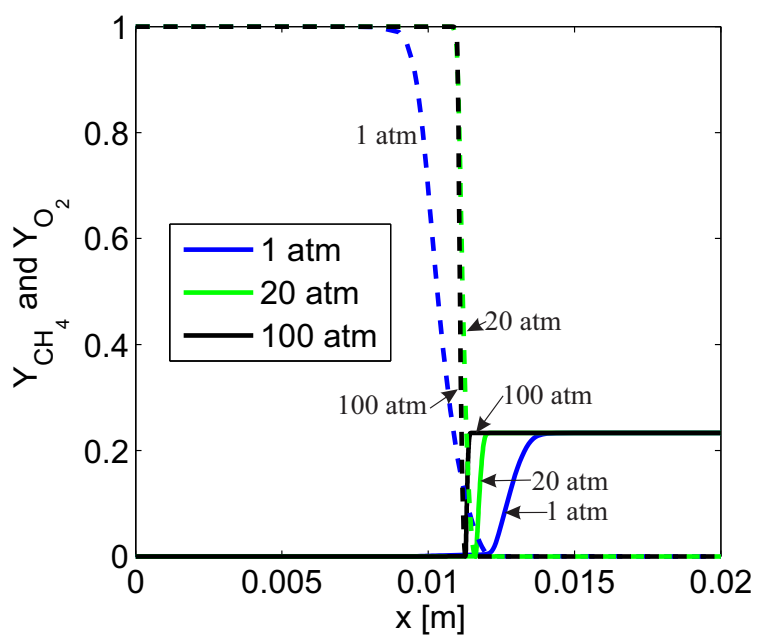

(c) Dashed: $Y_{\mathrm{CH}_{4}}$, Solid: $Y_{\mathrm{O}_{2}}$

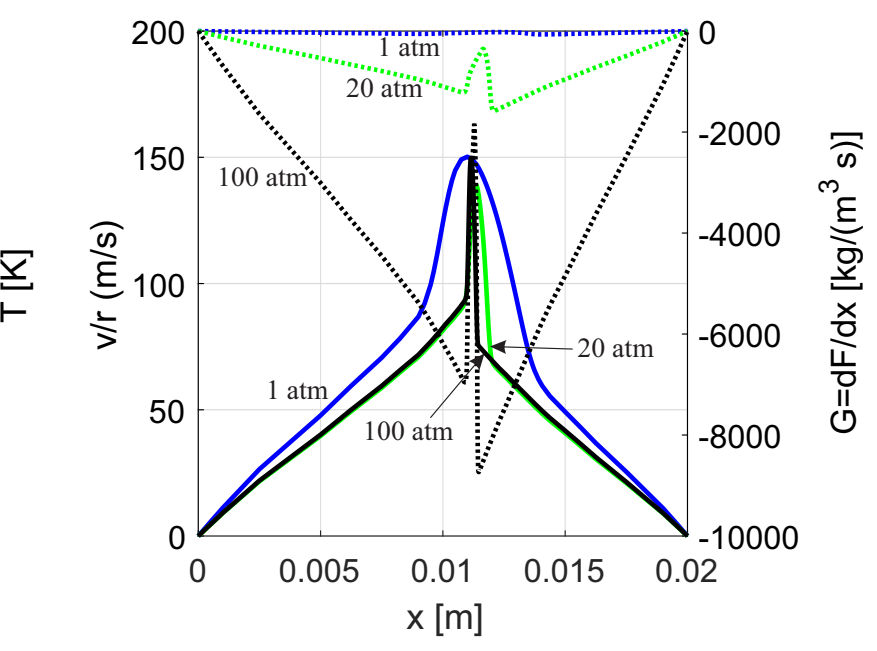

(b) Solid: $v / r$, Dotted: $G$

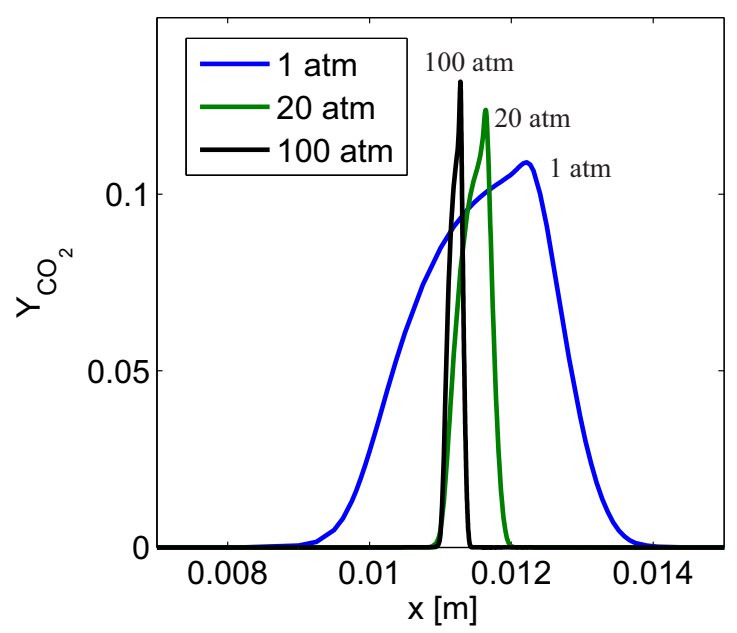

(d) $Y_{\mathrm{CO}_{2}}$

Figure 12: Set of variables for Case 6 at various pressures. 


\subsection{Case 8: Methane and Water Vapor}

Consider now Case 8 , in which water vapor flows into the domain premixed with the methane. Three sub-cases are studied with increasing amounts of water vapor: $10 \%, 20 \%$ and $40 \%$. For brevity, only a few representative plots are reported for the last case in figure 13 . The trends with increasing water content are discussed below.

At $10 \%$ water vapor, temperature profiles highlight that raising pressure yields identical effects as for previous cases: it increases flame temperature, it narrows flame width, and the temperature peak moves closer to the stagnation plane. Flame temperature at $1 \mathrm{~atm}$ is $2,029 \mathrm{~K}$ while it equals $2,305 \mathrm{~K}$ at $100 \mathrm{~atm}$. These values are $42 \mathrm{~K}$ and $49 \mathrm{~K}$ hotter than for Case 6, respectively. Note that the temperature boundary condition on the left side has been increased by $300 \mathrm{~K}$ with respect to all the previous cases. Thus, a new simulation of Case 6 where the left temperature boundary condition matches the $600 \mathrm{~K}$ of Case $8 \mathrm{a}$ is performed. The resulting flame temperature at $1 \mathrm{~atm}$ is 2,043 K. Now, Case $8 \mathrm{a}$ at $1 \mathrm{~atm}$ is $14 \mathrm{~K}$ colder compared to this new result. Therefore, addition of $10 \%$ of water acts as an energy sink. Nevertheless, $14 \mathrm{~K}$ is not a very substantial temperature difference. For enthalpy departure, the cold region of the air stream is unchanged with respect to Case 6 . The curves on the cold region of the fuel stream, however, are closer to each other, highlighting more sensitivity to the temperature boundary condition increment than to pressure.

Density is generally lower on the fuel side compared to Case 6 . The presence of water, which is heavier than methane, tends to increase the density. However, having a higher temperature on the left boundary is a dominant effect. Regarding the compressibility factor, the trend is inverted on the fuel side with respect to Case 6 . Here $(1-Z)$ is becoming more negative with increasing pressure, which also contributes to reduce the density.

Water product is generated in the flame region, where its mass fraction becomes greater than at the boundary for all pressures. The peak value increases with pressure. The previous trends on $\mathrm{CO}_{2}$ production do not seem to be altered with the presence of extra water. The hotter gas coming from the left enhances both $\mu$ and $\lambda$ on the fuel side, comparing with Case 6 . The properties are not altered in the rest of the domain.

The water content in the fuel mixture is elevated to $20 \%$ in Case $8 \mathrm{~b}$. The flame temperature is $21 \mathrm{~K}$ colder at $1 \mathrm{~atm}$ and $31 \mathrm{~K}$ colder at $100 \mathrm{~atm}$ compared to Case 8a. These flame temperatures are also lower than the case with no water. Again, the energy sink effect of water is dominant, and the consequence is even lower flame temperatures when more water is added. Density remains practically unaltered with respect to Case 8a. The presence of more water content on the left side of the domain affects the compressibility factor where all the $1-Z$ curves are moved in the positive direction, tending to increase the density of the mixture. Comparison against Case $8 \mathrm{a}$ indicates that the presence of extra water on the left stream increases the magnitude of the enthalpy departure function keeping the same sign in the cold zone on the fuel side. In the heat-release zone, the enthalpy departure behaves as in Case 8a.

Mass fraction of water features a local peak in the flame region, where more water is generated as a combustion product, but its magnitude now is lower than the 0.2 value coming from the left boundary. No changes occur in the $\mathrm{CO}_{2}$ mass fraction. Transport properties are also not altered. 


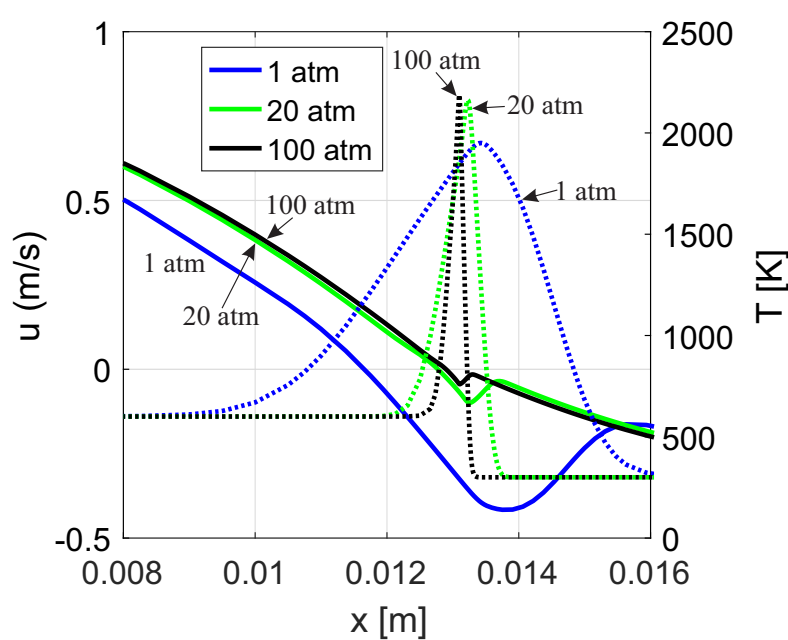

(a) Solid: $u$, Dotted: $T$

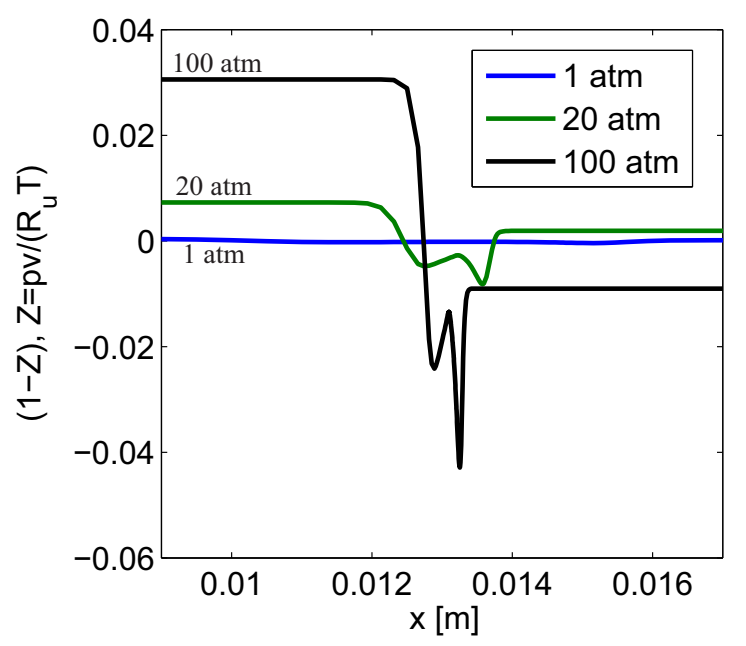

(c) $1-Z$

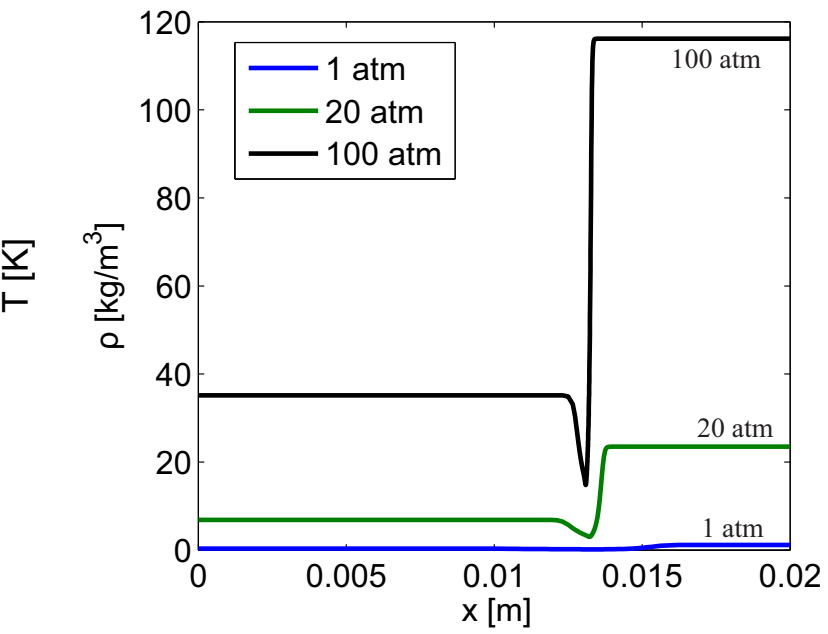

(b) $\rho$

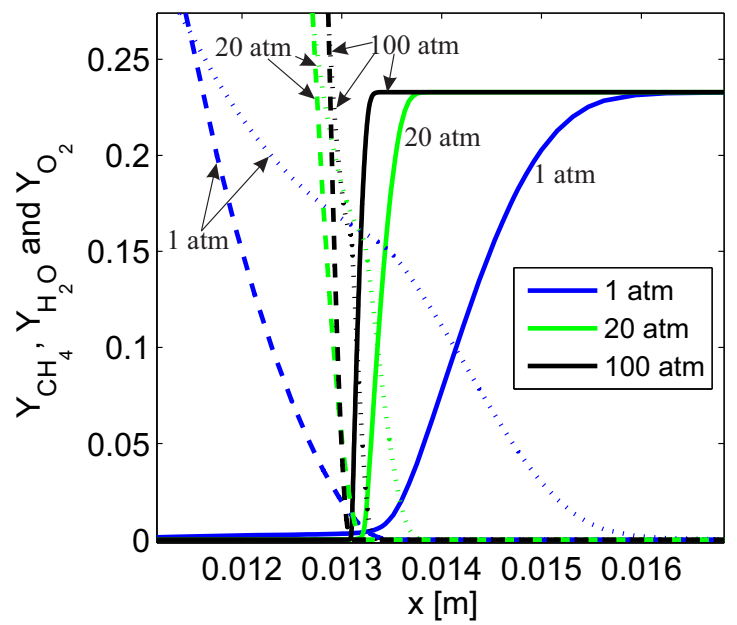

(d) Dashed: $Y_{\mathrm{CH}_{4}}$, Dots: $Y_{\mathrm{H}_{2} \mathrm{O}}$, Solid: $Y_{\mathrm{O}_{2}}$

Figure 13: Set of variables for Case $8 \mathrm{c}$ with $40 \%$ water vapor in the fuel stream. 
Figure 13 contains the solutions for Case $8 \mathrm{c}$ in which the water content in the fuel stream is $40 \%$. Similarly to all previous cases, the stagnation plane lies to the right of the symmetry plane. Peak temperature at 1 atm is $56 \mathrm{~K}$ colder comparing with Case $8 \mathrm{~b}$, and it is $90 \mathrm{~K}$ colder at $100 \mathrm{~atm}$. Density is shown in sub-figure $13 \mathrm{~b}$, portraying a slight increase with respect to Case $8 \mathrm{~b}$ on the cold fuel stream region. The $1-Z$ trend discussed for Case $8 \mathrm{~b}$ is even more obvious here as shown in sub-figure 13 , where all the curves are now positive at the left boundary.

Methane, oxygen, and water mass fractions are represented in Figure $13 \mathrm{~d}$. The water mass fraction flowing from the left is now too high compared to the water generated as a combustion product. This translates into a smooth decrease of the mass fraction gradient in the flame zone, which becomes steep again for greater $x$. The local peak that was identified in Cases $8 \mathrm{a}$ and $8 \mathrm{~b}$ no longer exists. The enthalpy departure function becomes more significant along the cold region of the fuel side, while it is diminished within the flame zone. Transport properties remain unaffected.

The water content in the fuel stream has been increased above $40 \%$ with the goal of finding the burning limit. The greatest percentage of $\mathrm{H}_{2} \mathrm{O}$ resulting in a flame solution is of $67 \%$ at 1 atm, with a flame temperature of $1,607 \mathrm{~K}$. Further study of this limit is required, including results at high pressure.

\section{Conclusion}

Analysis of combustion at high pressure with the presence of dense fluids requires relaxation of certain assumptions that are commonly taken. The use of ideal-gas approximations in these scenarios requires justification. This computational study presents solutions for a steady laminar counterflow diffusion flame using a real-gas model. The relative effect of different relaxations is analyzed by solving a one-dimensional system of differential equations equipped with detailed chemistry and detailed transport. The considered reactants are methane and air. A path for error estimates is identified in this canonical configuration. It can serve as a template for other researchers. In particular, three different corrections have been examined: (I) replacement of the ideal-gas law by a suitable non-ideal cubic equation of state; (II) consideration of the energy equation for real gases equipped with properly corrected thermodynamic properties such as enthalpy;

455 (III) modification of transport properties such as dynamic viscosity and thermal conductivity. Solutions are presented for several cases in which each of the corrections are applied individually or combined. Results show that the largest correction is the replacement of the ideal-gas law, followed by the amendment of the energy equation, while rectification of transport properties is less significant. Substantial differences appear mainly in terms of flame location. Correction of the equation of state moves the flame towards the

460 stagnation plane, closer to the fuel stream, whereas the correction of the energy equation has the opposite effect. The main differences in flame location are due to the fact that the real-gas equation of state provides more accurate values of the flow properties in the cold regions. This conclusion should be kept in mind during the design process of high-pressure combustors.

Even with the new corrections, heat-release rate, flame temperature, and thickness still follow well previously published correlations with pressure and pressure-weighed strain rate, respectively. Radiative heat losses and the Soret effect are shown to be negligible, even at the highest pressure.

An analysis has been developed on the existence of turbulence. Specifically, four plausible causes of turbulence in the counterflow have been identified, and based on established practice, we demonstrated that the first three mechanisms can essentially be eliminated. We showed, using an extremely conservative analysis (i.e., with elimination of turbulent kinetic energy diffusion and dissipation and with maximizing of the production term), that the fourth mechanism will not generate any significant turbulence for the applicable length scale.

The presented form of the energy equation is more general compared to the version commonly found in the literature, which assumes in the convective term that $h=\sum_{k} Y_{k} h_{k}$. Comparison of the two shows discrepancies in terms of flame temperature and enthalpy that are below $3 \%$.

The difference between matching mass fluxes versus momentum fluxes between the two nozzles affects only the flame location and does not does not impact any of our major findings. 
Furthermore, computations are made for a case equipped with the most complete model and including water vapor premixed with the methane. Solutions are presented for percentages of water vapor equal to $10 \%, 20 \%$ and $40 \%$ by mass. Presence of water not being generated as a combustion product acts as an energy sink, therefore flame temperatures decrease with increasing water content. Escalation of pressure produces similar effects on the flame for the problem with extra water and without it. These effects include increase of flame temperature, narrowing the flame region, and displacement of the flame position closer to the stagnation plane. This plane is located to the right of the symmetry line, closer to the air nozzle, for all the studied cases. The greatest percentage of premixed water content at which a flame solution has been obtained equals $67 \%$ at 1 atm. This limit requires further exploration, specially at higher pressures.

Commercial software packages use chemical kinetic laws established for lower pressure range. Chemical equilibrium laws at very high pressure require the use of fugacity rather than partial pressure. Comparative calculations have been presented for a range of prescribed temperatures and at 100 atm. Results indicate differences below 1\%. Thus, application of these kinetic laws at the higher pressures with regard to the ability to predict accurately equilibrium and rates becomes more comfortable. However, chemical pathways may still be different at high pressures.

\section{Acknowledgments}

This research was supported by the NSF under Grant CBET-1333605. The first author appreciates the Balsells Fellowship support.

\section{References}

[1] J. Foster, R. S. Miller, Fundamentals of high pressure, ProcessEng Engineering GmbH (2010) 53-75.

[2] T. Kamimoto, H. Kobayashi, Combustion processes in diesel engines, Prog. Eng. Comb. Sci. 17 (1991) $163-189$.

[3] R. D. Reitz, C. J. Rutland, Development and testing of diesel engine cfd models, Prog. Eng. Comb. Sci. 21 (1995) $173-196$.

[4] R. Marcer, P. L. Cottier, H. Chaves, B. Argueyrolles, C. Habchi, B. Barbey, A Validated Numerical Simulation of Diesel Injector Flow Using a VOF Method, Technical Report, SAE Paper 2000-01-2932, 2000.

[5] G. M. Bianchi, P. Pelloni, F. E. Corcione, L. Allocca, F. Luppino, Modeling atomization of high-pressure diesel sprays, J. Eng. Gas Turb. Power 123 (2001) 419-427.

[6] E. de Villiers, A. D. Gosman, H. G. Weller, Large Eddy Simulation of Primary Diesel Spray Atomization, Technical Report, SAE Paper 2004-01-0100, 2004.

[7] J. P. Delplanque, W. A. Sirignano, Numerical study of the transient vaporization of an oxygen droplet at sub- and super-critical conditions, Int. J. Heat Mass Transfer 36 (1993) 303-314.

[8] W. Mayer, H. Tamura, Propellant injection in a liquid oxygen/gaseous hydrogen rocket engine, J. Propul. Power 12 (1996) $1137-1147$.

[9] J. P. Delplanque, W. A. Sirignano, Transcritical liquid oxigen droplet vaporization: Effect on rocket combustion instability, J. Propul. Power 12 (1996) 349-357.

[10] L. Pons, N. Darabiha, S. Candel, G. Ribert, V. Yang, Mass transfer and combustion in transcritical non-premixed counterflows, Combust. Theor. Model. 13 (2009) 57-81.

[11] E. D. Sloan, C. A. Koh, Clathrate Hydrates of Natural Gases. 3rd Edition, CRC Press Taylor and Francis, 2007.

[12] K. A. Kvenvolden, Methane hydrate - a major reservoir of carbon in the shallow geosphere?, Chem. Geol. 71 (1988) $41-51$.

[13] T. Bar-Kohany, W. A. Sirignano, Transient combustion of a methane-hydrate sphere, Combust. Flame (2015).

[14] C. K. Law, Combustion Physics, Cambridge University Press, Cambridge, UK, 2006.

[15] F. A. Williams, Combustion Theory. 2nd Edition, The Benjamin/Cummings Publishing Company, Inc., Menlo Park, CA, 1985.

[16] S. R. Turns, An Introduction to Combustion: Concepts and Applications. 3rd Edition, McGraw Hill, 2011.

[17] F. E. Marble, J. E. Broadwell, The Coherent Flame Model for Turbulent Chemical Reactions, Technical Report, Project SQUID, Report TRW-9-PU, 1977.

[18] G. Ribert, N. Zong, V. Yang, L. Pons, N. Darabiha, S. Candel, Counterflow diffusion flames of general fluids: Oxygen/hydrogen mixtures, Combust. Flame 154 (2008) 319-330.

[19] G. Lacaze, J. C. Oefelein, A non-premixed combustion model based on flame structure analysis at supercritical pressures, Combust. Flame 159 (2012) 2087-2103.

[20] H. Huo, X. Wang, V. Yang, A general study of counterflow diffusion flames at subcritical and supercritical conditions: Oxygen/hydrogen mixtures, Combust. Flame 161 (2014) 3040-3050.

[21] X. Wang, H. Huo, V. Yang, Counterflow diffusion flames of oxygen and n-alkane hydrocarbons $\left(\mathrm{CH}_{4}-\mathrm{C}_{16} \mathrm{H}_{34}\right)$ at subcritical and supercritical conditions, Combust. Sci. Technol. 187 (2015) 60-82. 
[22] G. Amantini, J. H. Frank, M. D. Smooke, A. Gomez, Computational and experimental study of steady axisymmetric non-premixed methane counterflow flames, Combust. Theor. Model. 11 (2007) 47-72.

[23] U. Niemann, K. Seshadri, F. A. Williams, Methane, ethane, and ethylene laminar counterflow diffusion flames at elevated pressures: Experimental and computational investigations up to 2.0 mpa, Combust. Flame 161 (2014) $138-146$.

[24] L. Piller, M. Idir, J. Molet, A. Matynia, S. de Persis, Experimental study and modelling of nox formation in high pressure counter-flow premixed $\mathrm{CH}_{4}$ /air flames, Fuel 150 (2015) 394-407.

[25] L. Figura, F. Carbone, A. Gomez, Challenges and artifacts of probing high-pressure counterflow laminar diffusion flames, Proc. Combust. Inst. 35 (2015) 1871-1878.

[26] A. Abbud-Madrid, E. P. Riedel, J. T. McKinnon, The influence of water mists on premixed flame propagation in microgravity, Symp. (Int.) Micrograv. Res. Appl. Phys. Sci. Biotech. 1 (2000) 313-320.

[27] V. Ricchiuti, R. E. Padilla, S.Karnani, D. Dunn-Rankin, Cantera simulations of water-laden methane/air nonpremixed counterflow flames, 8th US Nat. Comb. Meeting (2013).

[28] V. Giovangigli, L. Matuszewski, Numerical simulation of transcritical strained laminar flames, Combust. Flame 159 (2012) 2829-2840.

[29] M. I. Hassan, A. T. Brimmo, Modeling in-cylinder water injection in a 2-stroke internal combustion engine, Energy Procedia 75 (2015) 2331-2336.

[30] A. Yoshida, R. Takasaki, K. Kashiwa, H. Naitob, Y. Saso, Extinguishment of counterflow methane/air diffusion flame by polydisperse fine water droplets, Combust. Flame 160 (2013) 1357-1363.

[31] J. P. Wilson, Effects of Water Injection and Increased Compression Ratio in a Gasoline Spark Ignition Engine, Ph.D. thesis, University of Idaho, Idaho, 2011.

[32] D. Liu, A. M. Zghoul, Z. Li, B. Tan, K. L. Lockwood, R. H. Essenhigh, High intensity combustion of coal with water injection, Combust. Flame 63 (1986) 49-57.

[33] M. D. Smooke, I. K. Puri, K. Seshadri, A comparison between numerical calculations and experimental measurments of the structure of a counterflow diffusion flame burning diluted methane in diluted air, Symp. (Int.) Combust. (1986) 1783-1792.

[34] R. J. Kee, J. A. Miller, G. H. Evans, A computational model of the structure and extinction of strained, opposed flow, premixed methane-air flames, Symp. (Int.) Combust. (1988) 1479-1494

[35] T. Chung, M. Ajlan, L. L. Lee, K. E. Starling, Generalized multiparameter correlation for nonpolar and polar fluid transport properties, Ind. Eng. Chem. Res. 27 (1988) 671-679.

[36] Chemkin 10131, Reaction Design, San Diego, 2013.

[37] U. Niemann, K. Seshadri, F. A. Williams, Accuracies of laminar counterflow flame experiments, Combust. Flame 162 (2015) 1540-1549.

[38] G. P. Smith, D. M. Golden, M. Frenklach, N. W. Moriarty, B. Eiteneer, M. Goldenberg, C. T. Bowman, R. K. Hanson, S. Song, W. C. G. Jr., V. V. Lissianski, Z. Qin, Gri-mech 3.0, http://www.me.berkeley.edu/gri_mech/, 2000.

[39] G. Soave, Equilibrium constants from a modified redlich-kwong equation of state, Chem. Eng. Sci. 27 (1972) $1197-1203$.

[40] G. Soave, S. Gamba, L. A. Pellegrini, Srk equation of state: Predicting binary interaction parameters of hydrocarbons and related compounds, Fluid Phase Equilib. 299 (2010) 285-293.

[41] J. M. Prausnitz, R. N. Lichtenthaler, E. G. de Azevedo, Molecular Thermodynamics of Fluid-Phase Equilibria, Prentice Hall PTR, Englewood Cliffs, N.J., 1999.

[42] V. Giovangigli, L. Matuszewski, F. Dupoirieux, Detailed modeling of planar transcritical $\mathrm{H}_{2}-\mathrm{O}_{2}-\mathrm{N}_{2}$ flames, Combust. Theor. Model. 15 (2011) 141-182.

[43] Y. V. C. Rao, Chemical Engineering Thermodynamics, Universities Press, 1997.

[44] J. F. Grcar, The Twopnt Program for Boundary Value Problems, Technical Report, Sandia National Laboratories Report SAND91-8230, 1992.

[45] T. Lu, C. K. Law, A criterion based on computational singular perturbation for the identification of quasi steady state species: A reduced mechanism for methane oxidation with no chemistry, Combust. Flame. 154 (2008) $761-774$.

[46] M. Chmielewski, M. Gieras, Planck mean absorption coefficients of $\mathrm{H}_{2} \mathrm{O}, \mathrm{CO}_{2}$, $\mathrm{CO}$ and $\mathrm{NO}$ for radiation numerical modeling in combusting flows, J. Power Technologies 95 (2015) 97-104.

[47] B. E. Launder, D. B. Spalding, Lectures in Mathematical Models of Turbulence, Academic Press Inc., New York, N.Y., 1972 .

[48] L. Figura, A. Gomez, Laminar counterflow steady diffusion flames under high pressure $(p \leq 3$ mpa) conditions, Combust. Flame 159 (2012) 142-150.

[49] R. Holub, P. Voňka, The Chemical Equilibrium of Gaseous Systems, Springer Netherlands, 1976. 


\section{Appendix A. Chemical Equilibrium}

Reaction mechanisms provide data to compute the forward rates of each reaction that is part of the mechanism. Reverse rates are usually computed from forward rates and the principle of chemical equilibrium. This principle is embedded in most commercial codes, usually based on the classical partial pressures. At high pressure, however, fugacities should be used instead of partial pressures for better accuracy in chemicalequilibrium calculation. The theory for chemical kinetics of non-ideal gases is not well established. So, we are forced to use the same kinetics developed for ideal gases. The goal of this section is to assess the differences that arise when we calculate the product species composition in equilibrium of a hydrocarbon reaction, both using the classical ideal formulation and the non-ideal counterpart.

In the following, the index $i$ corresponds to a particular reaction while the index $k$ indicates species. $\nu_{k i}$ represents the stoichiometric coefficient of species $k$ in the reaction $i . X^{\text {classic }}$ and $X$ are the mole fractions of the product species in chemical equilibrium for the classical case and for the non-ideal case, respectively.

The equilibrium constant is determined from thermodynamics:

$$
K_{p_{i}}(T)=\exp \left(-\frac{\Delta G_{i}^{o}}{R_{u} T}\right)=\exp \left(\frac{\Delta S_{i}^{o}}{R_{u}}-\frac{\Delta H_{i}^{o}}{R_{u} T}\right)
$$

where $\Delta G_{i}^{o}, \Delta H_{i}^{o}$, and $\Delta S_{i}^{o}$ are the Gibbs function, enthalpy, and entropy changes that occur in passing completely from reactants to products in the $i$ th reaction.

In the classical case, the equilibrium constant for a mixture of ideal gases is based on partial pressures:

$$
K_{p_{i}}=\frac{\prod_{k}\left(P_{k} / P^{o}\right)^{\nu_{k i}^{\text {products }}}}{\prod_{k}\left(P_{k} / P^{o}\right)^{\nu_{k i}^{\text {reactants }}}}=\frac{\prod_{k}\left(X_{k}^{\text {classical }}\right)^{\nu_{k i}^{\text {products }}}}{\prod_{k}\left(X_{k}^{\text {classical }}\right)^{\nu_{k i}^{\text {reactants }}}}\left(\frac{P}{P^{o}}\right)^{\Delta \nu_{i}}
$$

where $P^{o}$ is the standard state pressure of 1 atm, and $\Delta \nu_{i}=\sum_{k} \nu_{k i}^{\text {products }}-\sum_{k} \nu_{k i}^{\text {reactants }}$.

For non-ideal systems, fugacities must be employed [49]:

$$
K_{p_{i}}=\frac{\prod_{k}\left(f_{k} / P^{o}\right)^{\nu_{k i}^{\text {products }}}}{\prod_{k}\left(f_{k} / P^{o}\right)^{\nu_{k i}^{\text {reactants }}}}=\frac{\prod_{k}\left(\phi_{k} X_{k}\right)^{\nu_{k i}^{\text {products }}}}{\prod_{k}\left(\phi_{k} X_{k}\right)^{\nu_{k i}^{\text {reactants }}}}\left(\frac{P}{P^{o}}\right)^{\Delta \nu_{i}}
$$

where $f_{k}$ is the fugacity of species $k$ and $\phi_{k}=f_{k} /\left(X_{k} P\right)$ is the fugacity coefficient. These expressions for both classical and non-ideal cases will be used in the laws of mass action below.

A generic reaction between a hydrocarbon and air is

$$
\begin{aligned}
n_{F}\left[\mathrm{C}_{n} \mathrm{H}_{m}+\frac{n+m / 4}{\Phi}\left(\mathrm{O}_{2}+3.76 \mathrm{~N}_{2}\right)\right] \longrightarrow & \\
X_{1} \mathrm{O}_{2}+X_{2} \mathrm{CO}_{2}+ & X_{3} \mathrm{H}_{2} \mathrm{O}+X_{4} \mathrm{CO}+X_{5} \mathrm{OH} \\
& +X_{6} \mathrm{H}+X_{7} \mathrm{H}_{2}+X_{8} \mathrm{O}+X_{9} \mathrm{~N}+X_{10} \mathrm{~N}_{2}+X_{11} \mathrm{NO}
\end{aligned}
$$

where $n_{F}$ is the number of moles that gives one mole of products, $X_{1}$ through $X_{11}$ are the mole fractions of the considered product species, and $\Phi$ is the fuel-to-air equivalence ratio.

To determine the mass fractions $X_{k}(k=1, \ldots, 11)$ and $n_{F}$ at equilibrium, we use four mass balance equations, seven laws of mass action associated with elementary reactions, and the fact that the summation of all mass fractions must equal one. In the following, $r=\frac{n+m / 4}{\Phi}$.

$$
\left\{\begin{array}{l}
n n_{F}=X_{2}+X_{4} \\
m n_{F}=2 X_{3}+X_{5}+X_{6}+2 X_{7} \\
2 r n_{F}=2 X_{1}+2 X_{2}+X_{3}+X_{4}+X_{5}+X_{8}+X_{11} \\
7.52 r n_{F}=X_{9}+2 X_{10}+X_{11}
\end{array}\right.
$$


Table A.2: $X_{k}^{\text {classic }} / X_{k}$, Stoichiometric: $\Phi=1.0$

\begin{tabular}{|c|c|c|c|c|c|c|c|c|c|c|c|}
\hline $\mathrm{T}(\mathrm{K})$ & $\mathrm{O}_{2}$ & $\mathrm{CO}_{2}$ & $\mathrm{H}_{2} \mathrm{O}$ & $\mathrm{CO}$ & $\mathrm{OH}$ & $\mathrm{H}$ & $\mathrm{H}_{2}$ & $\mathrm{O}$ & $\mathrm{N}$ & $\mathrm{N}_{2}$ & $\mathrm{NO}$ \\
\hline 2300 & 1.00569 & 0.99992 & 0.99995 & 1.00267 & 1.00164 & 1.00013 & 1.00660 & 1.00291 & 1.00236 & 0.99999 & 1.00037 \\
\hline 2400 & 1.00523 & 0.99988 & 0.99994 & 1.00260 & 1.00141 & 1.00012 & 1.00626 & 1.00268 & 1.00227 & 0.99998 & 1.00024 \\
\hline 2500 & 1.00484 & 0.99984 & 0.99992 & 1.00251 & 1.00122 & 1.00010 & 1.00594 & 1.00248 & 1.00219 & 0.99997 & 1.00014 \\
\hline 2600 & 1.00452 & 0.99978 & 0.99989 & 1.00242 & 1.00109 & 1.00011 & 1.00570 & 1.00232 & 1.00212 & 0.99997 & 1.00007 \\
\hline 2700 & 1.00434 & 0.99970 & 0.99985 & 1.00236 & 1.00123 & 1.00028 & 1.00580 & 1.00230 & 1.00207 & 0.99996 & 1.00005 \\
\hline 2800 & 1.00417 & 0.99961 & 0.99980 & 1.00229 & 1.00134 & 1.00043 & 1.00585 & 1.00229 & 1.00204 & 0.99994 & 1.00002 \\
\hline 2900 & 1.00401 & 0.99950 & 0.99975 & 1.00220 & 1.00141 & 1.00056 & 1.00587 & 1.00227 & 1.00200 & 0.99993 & 1.00000 \\
\hline 3000 & 1.00384 & 0.99937 & 0.99968 & 1.00209 & 1.00145 & 1.00066 & 1.00585 & 1.00224 & 1.00198 & 0.99991 & 0.99997 \\
\hline
\end{tabular}

The elementary reactions and their corresponding laws of mass action (based on partial pressures) are

$$
\begin{cases}\frac{1}{2} \mathrm{O}_{2} \rightleftarrows \mathrm{O}, & K_{p_{1}}=\frac{X_{8}}{X_{1}^{1 / 2}}\left(\frac{P}{P_{o}}\right)^{1 / 2} \\ \frac{1}{2} \mathrm{H}_{2} \rightleftarrows \mathrm{H}, & K_{p_{2}}=\frac{X_{6}}{X_{7}^{1 / 2}}\left(\frac{P}{P_{o}}\right)^{1 / 2} \\ \frac{1}{2} \mathrm{~N}_{2} \rightleftarrows \mathrm{N}, & K_{p_{3}}=\frac{X_{9}}{X_{10}^{1 / 2}}\left(\frac{P}{P_{o}}\right)^{1 / 2} \\ \frac{1}{2} \mathrm{O}_{2}+\frac{1}{2} \mathrm{~N}_{2} \rightleftarrows \mathrm{NO}, & K_{p_{4}}=\frac{X_{11}}{X_{1}^{1 / 2} X_{10}^{1 / 2}} \\ \mathrm{CO}+\frac{1}{2} \mathrm{O}_{2} \rightleftarrows \mathrm{CO}_{2}, & K_{p_{5}}=\frac{X_{2}}{X_{4} X_{1}^{1 / 2}}\left(\frac{P}{P_{o}}\right)^{-1 / 2} \\ \mathrm{H}_{2}+\frac{1}{2} \mathrm{O}_{2} \rightleftarrows \mathrm{H}_{2} \mathrm{O}, & K_{p_{6}}=\frac{X_{3}}{X_{7} X_{1}^{1 / 2}}\left(\frac{P}{P_{o}}\right)^{-1 / 2} \\ \frac{1}{2} \mathrm{H}_{2}+\frac{1}{2} \mathrm{O}_{2} \rightleftarrows \mathrm{OH}, & K_{p_{7}}=\frac{X_{5}}{X_{7}^{1 / 2} X_{1}^{1 / 2}}\end{cases}
$$

These laws of mass action are readily modified for the non-ideal case by multiplying each mass fraction by its fugacity coefficient, according to Equation A.3. The fugacity coefficients are obtained according to our choice of EoS, from the following expression [39]:

$$
\ln \left(\phi_{k}\right)=\frac{b_{k}}{b}(Z-1)-\ln (Z-B)-\frac{A}{B}\left(2 \frac{a_{k}^{0.5}}{a^{0.5}}-\frac{b_{k}}{b}\right) \ln \left(1+\frac{B}{Z}\right)
$$

Equations A.5 and A.6. together with $\sum_{k=1}^{11} X_{k}=1$, form a system of non-linear algebraic equations. We use the Matlab solver "fsolve", together with the "trust-region-dogleg" algorithm. The pressure is prescribed at $100 \mathrm{~atm}$, and the temperature is varied from $2300 \mathrm{~K}$ to $3000 \mathrm{~K}$. The product composition is obtained for three different equivalence ratios $(\Phi=1,0.8$, and 1.1). Ratios between the mole fractions for the classical case and those for the non-ideal case are formed and presented in Tables A.2, A.3, and A.4.

We can see from the results that the differences are very small, always below $1 \%$ for the stoichiometric and fuel-lean cases . Only the fuel-rich case shows ratios slightly above $1 \%$, but it only exceeds this value by a few decimals. These results make the use of the classical chemical equilibrium more comfortable at elevated pressures for combustion problems. However, one must keep in mind that differences in chemical pathways may still exist, and therefore the uncertainty on reaction rates also remains. 
Table A.3: $X_{k}^{\text {classic }} / X_{k}$, Fuel-lean: $\Phi=0.8$

\begin{tabular}{|l|c|c|c|c|c|c|c|c|c|c|c|}
\hline $\mathrm{T}(\mathrm{K})$ & $\mathrm{O}_{2}$ & $\mathrm{CO}_{2}$ & $\mathrm{H}_{2} \mathrm{O}$ & $\mathrm{CO}$ & $\mathrm{OH}$ & $\mathrm{H}$ & $\mathrm{H}_{2}$ & $\mathrm{O}$ & $\mathrm{N}$ & $\mathrm{N}_{2}$ & $\mathrm{NO}$ \\
\hline 2300 & 1.00022 & 0.99997 & 0.99999 & 1.00556 & 1.00050 & 1.00168 & 1.00974 & 1.00023 & 1.00240 & 1.00003 & 0.99766 \\
\hline 2400 & 1.00024 & 0.99995 & 0.99998 & 1.00528 & 1.00042 & 1.00158 & 1.00921 & 1.00024 & 1.00232 & 1.00003 & 0.99777 \\
\hline 2500 & 1.00027 & 0.99992 & 0.99997 & 1.00501 & 1.00035 & 1.00148 & 1.00871 & 1.00026 & 1.00224 & 1.00002 & 0.99788 \\
\hline 2600 & 1.00031 & 0.99988 & 0.99995 & 1.00475 & 1.00032 & 1.00141 & 1.00831 & 1.00029 & 1.00217 & 1.00002 & 0.99798 \\
\hline 2700 & 1.00037 & 0.99982 & 0.99993 & 1.00458 & 1.00050 & 1.00151 & 1.00825 & 1.00039 & 1.00213 & 1.00001 & 0.99808 \\
\hline 2800 & 1.00045 & 0.99973 & 0.99989 & 1.00438 & 1.00065 & 1.00159 & 1.00815 & 1.00049 & 1.00210 & 1.00000 & 0.99819 \\
\hline 2900 & 1.00055 & 0.99962 & 0.99984 & 1.00415 & 1.00077 & 1.00164 & 1.00801 & 1.00060 & 1.00207 & 0.99998 & 0.99829 \\
\hline 3000 & 1.00065 & 0.99948 & 0.99978 & 1.00389 & 1.00087 & 1.00167 & 1.00783 & 1.00071 & 1.00204 & 0.99996 & 0.99840 \\
\hline
\end{tabular}

Table A.4: $X_{k}^{\text {classic }} / X_{k}$, Fuel-rich: $\Phi=1.1$

\begin{tabular}{|c|c|c|c|c|c|c|c|c|c|c|c|}
\hline $\mathrm{T}(\mathrm{K})$ & $\mathrm{O}_{2}$ & $\mathrm{CO}_{2}$ & $\mathrm{H}_{2} \mathrm{O}$ & $\mathrm{CO}$ & $\mathrm{OH}$ & $\mathrm{H}$ & $\mathrm{H}_{2}$ & $\mathrm{O}$ & $\mathrm{N}$ & $\mathrm{N}_{2}$ & $\mathrm{NO}$ \\
\hline 2300 & 1.01385 & 1.00034 & 0.99986 & 0.99900 & 1.00353 & 0.99799 & 1.00230 & 1.00695 & 1.00236 & 1.00000 & 1.00443 \\
\hline 2400 & 1.01298 & 1.00030 & 0.99987 & 0.99913 & 1.00321 & 0.99809 & 1.00218 & 1.00651 & 1.00228 & 1.00000 & 1.00410 \\
\hline 2500 & 1.01216 & 1.00026 & 0.99987 & 0.99926 & 1.00291 & 0.99819 & 1.00209 & 1.00610 & 1.00220 & 0.99999 & 1.00379 \\
\hline 2600 & 1.01139 & 1.00021 & 0.99987 & 0.99939 & 1.00268 & 0.99831 & 1.00208 & 1.00572 & 1.00212 & 0.99999 & 1.00350 \\
\hline 2700 & 1.01092 & 1.00017 & 0.99984 & 0.99951 & 1.00275 & 0.99855 & 1.00233 & 1.00555 & 1.00207 & 0.99998 & 1.00333 \\
\hline 2800 & 1.01024 & 1.00009 & 0.99980 & 0.99970 & 1.00273 & 0.99883 & 1.00264 & 1.00527 & 1.00203 & 0.99997 & 1.00306 \\
\hline 2900 & 1.00937 & 0.99997 & 0.99974 & 0.99994 & 1.00264 & 0.99913 & 1.00301 & 1.00491 & 1.00200 & 0.99995 & 1.00268 \\
\hline 3000 & 1.00841 & 0.99981 & 0.99967 & 1.00020 & 1.00249 & 0.99943 & 1.00340 & 1.00449 & 1.00197 & 0.99993 & 1.00225 \\
\hline
\end{tabular}

\section{Appendix B. Mass Flux vs Momentum Flux}

The differences between the boundary conditions for $F$ introduced by Equation 10 (matching the mass fluxes of both nozzles), versus matching the momentum fluxes are highlighted in Figure B.14. It includes velocity, temperature, density, and mass fraction curves for Case 6 at various pressures. We observe that the main difference between the two boundary conditions is a shift in the $\mathrm{x}$-coordinate of the flame and the stagnation plane. Finding the stagnation plane in the center of the domain is expected when balancing the momentum fluxes. This clearly occurs at elevated pressures. At atmospheric pressure, however, the heat release zone is wide enough to perturb the velocity field and cause stagnation to occur closer to the fuel side. 


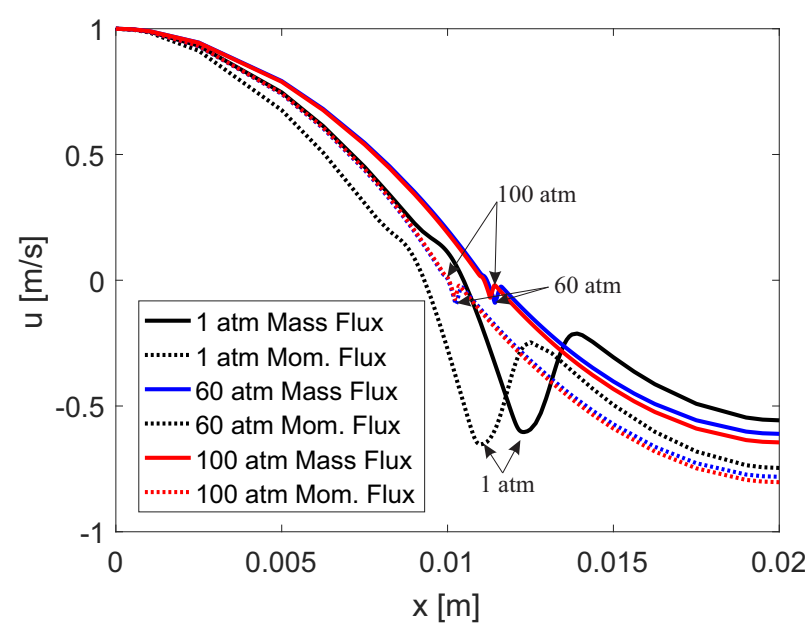

(a) $u$

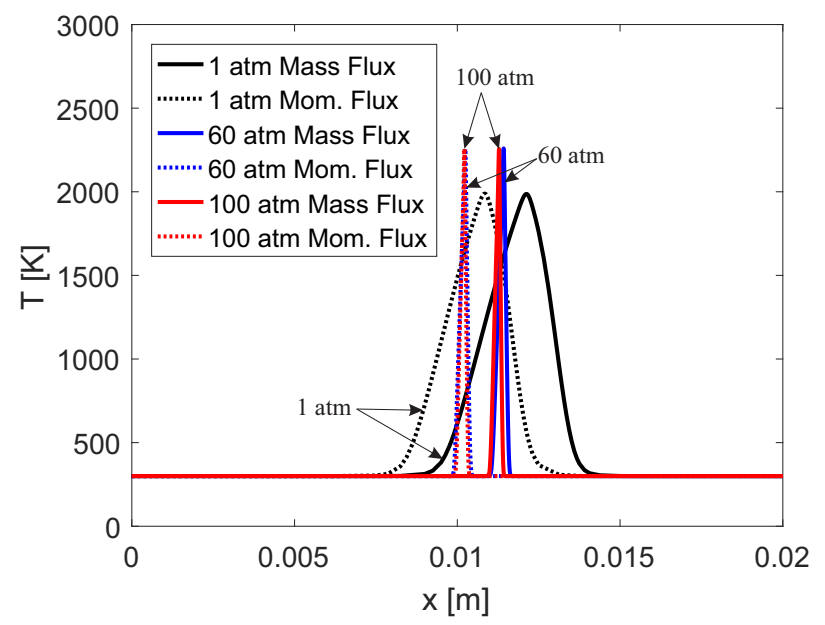

(c) $T$

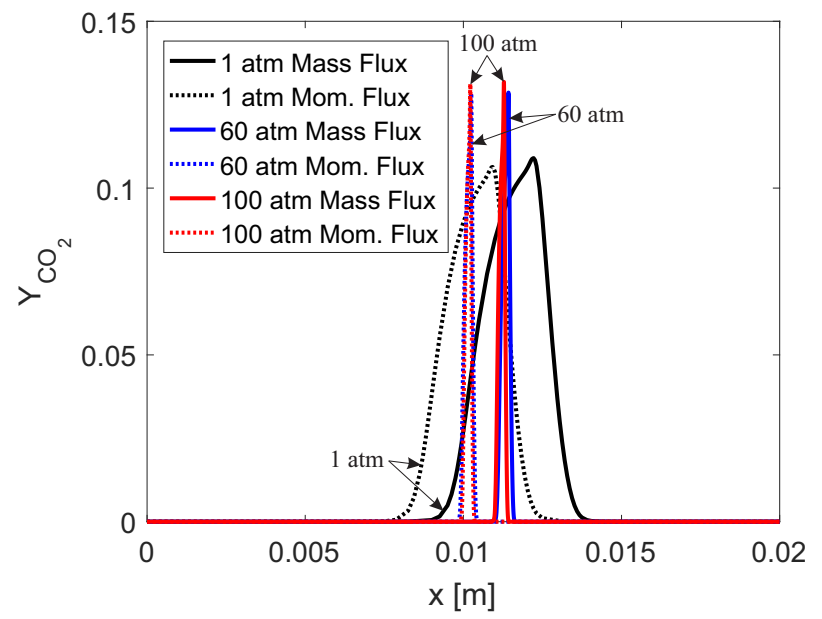

(e) $Y_{\mathrm{CO}_{2}}$

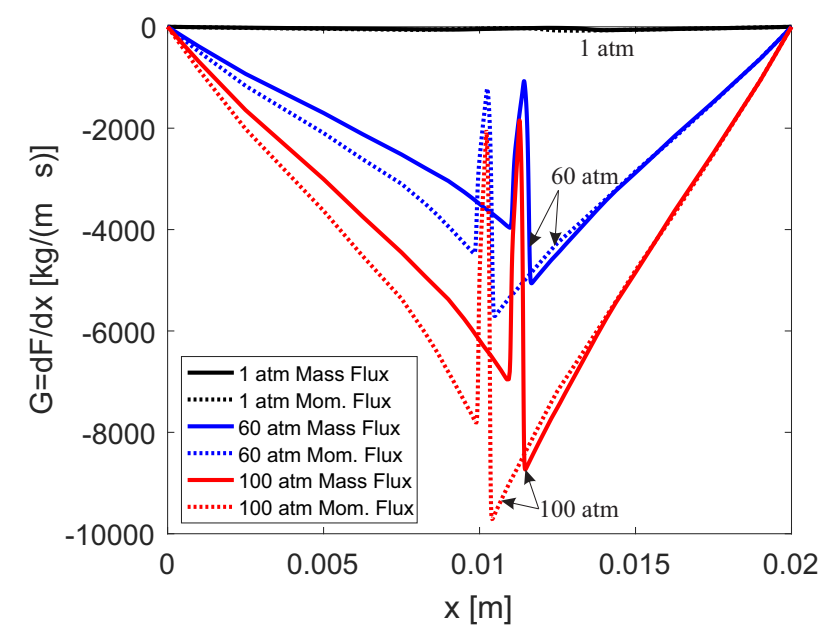

(b) $G$

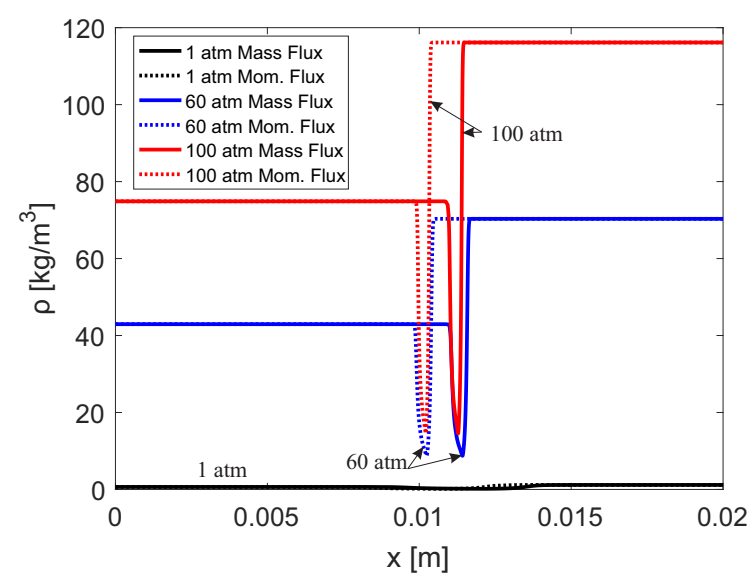

(d) $\rho$

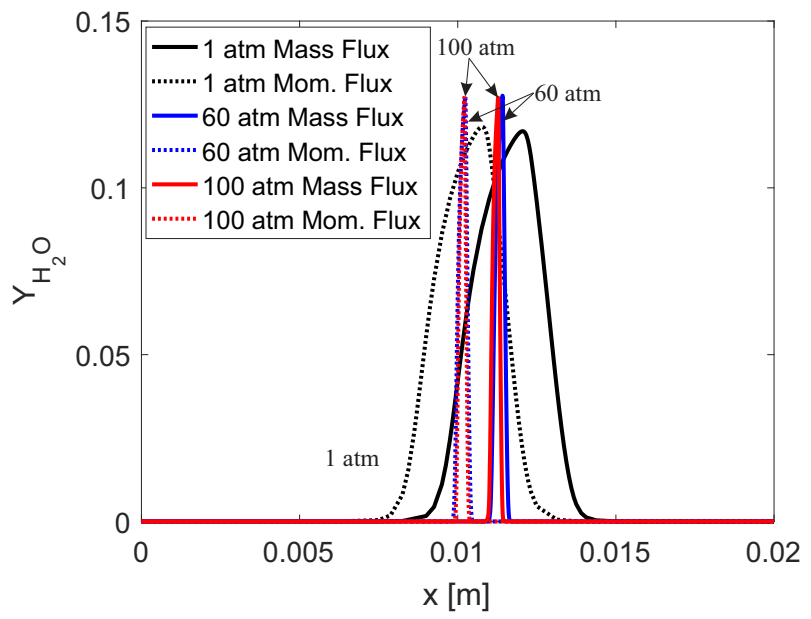

(f) $Y_{\mathrm{H}_{2}} \mathrm{O}$

Figure B.14: Comparison between matching mass fluxes versus matching momentum fluxes for Case 6 . 